\title{
A NEW APPROACH TO THE LENNARD-JONES POTENTIAL AND A NEW MODEL: PNP-STERIC EQUATIONS*
}

\author{
TAI-CHIA LIN ${ }^{\dagger}$ AND BOB EISENBERG ${ }^{\ddagger}$
}

\begin{abstract}
A class of approximate Lennard-Jones (LJ) potentials with a small parameter is found whose Fourier transforms have a simple asymptotic behavior as the parameter goes to zero. When the LJ potential is replaced by the approximate LJ potential, the total energy functional becomes simple and exactly the same as replacing the LJ potential by a delta function. Such a simple energy functional can be used to derive the Poisson-Nernst-Planck equations with steric effects (PNP-steric equations), a new mathematical model for the LJ interaction in ionic solutions. Using formal asymptotic analysis, stability and instability conditions for the 1D PNP-steric equations with the Dirichlet boundary conditions for one anionic and cationic species are expressed by the valences, diffusion constants, ionic radii, and coupling constants. This is the first step to study the dynamics of solutions of the PNP-steric equations.
\end{abstract}

Key words. Lennard-Jones potentials, Poisson-Nernst-Planck equations, steric effects.

AMS subject classifications. 35B35, 35K57, 92C05.

\section{Introduction}

The Lennard-Jones (LJ) potential, a well-known mathematical model for the interaction between a pair of ions, has important applications in many fields of biology, chemistry and physics (cf. [46]). Such a capable model can be represented by

$$
\Psi(x)=\frac{C_{1}}{r^{12}}-\frac{C_{2}}{r^{6}} \quad \text { for } \quad r=|x|>0, \quad x \in \mathbb{R}^{d},
$$

where $C_{1}, C_{2}$ are positive constants related to finite ion size, and $d \leq 3$ is the spatial dimension (cf. [36]). The inverse twelfth-power term is the repulsive term of $\Psi$ and the inverse sixth-power term is the attractive term of $\Psi$. The LJ potential $\Psi$ can be extended to an $l-m$ LJ potential given as follows:

$$
\Psi_{l, m}(x)=\frac{C_{1}}{r^{m}}-\frac{C_{2}}{r^{l}} \quad \text { for } \quad r=|x|>0, \quad x \in \mathbb{R}^{d},
$$

where $l$ and $m$ are any positive constants with $m>l>d$. All the mathematical arguments of the LJ potential $\Psi$ here can easily be generalized to the $l-m$ LJ potential for $m>l>d, C_{1}>0$, and $C_{2} \geq 0$.

To compute the energy of ions interacting by the LJ potential, the following energy functional is considered:

$$
E_{L J}\left[c_{i}, c_{j}\right]=\iint_{\mathbb{R}^{d} \times \mathbb{R}^{d}} \Psi(x-y) c_{i}(x) c_{j}(y) d x d y,
$$

for nonnegative functions $c_{i}$ and $c_{j}$ which denote the distribution (concentration) functions of the $i$ th and $j$ th ion species (cf. [29]). Similar energy functionals for Coulomb interactions can be found in [9]. Note that the energy functional $E_{L J}$ is for

*Received: September 4, 2012; accepted (in revised form): February 5, 2013. Communicated by Amit Singer.

${ }^{\dagger}$ Department of Mathematics, National Taiwan University, Taida Institute for Mathematical Sciences (TIMS), No.1, Sec.4, Roosevelt Road, Taipei 106, Taiwan (tclin@math.ntu.edu.tw).

$\ddagger$ Department of Molecular Biophysics \& Physiology Rush Medical Center, 1653 West Congress, Parkway, Chicago, IL 60612, USA (beisenbe@rush.edu). 
two ion species if $i \neq j$, but for only one ion species if $i=j$. Because the LJ potential $\Psi$ is singular at the origin, the functional $E_{L J}$ is a singular integral defined by

$$
E_{L J}\left[c_{i}, c_{j}\right]=\lim _{\sigma \rightarrow 0} \iint_{\mathbb{R}^{d} \times \mathbb{R}^{d}} \Psi \chi_{\sigma}(x-y) c_{i}(x) c_{j}(y) d x d y,
$$

for $c_{i}, c_{j} \in L_{+}^{2}\left(\mathbb{R}^{d}\right)=\left\{f \in L^{2}\left(\mathbb{R}^{d}\right): f \geq 0\right\}$, where $\chi_{\sigma}=\chi_{\sigma}(z)$ is the characteristic function of the exterior ball $\left\{z \in \mathbb{R}^{d}:|z|>\sigma\right\}$. In $[16,17,18,28]$, the energy functional $E_{L J}$ with $C_{2}=0$ is used, but here both $C_{2}=0$ and $C_{2}>0$ are considered.

Conventionally, the system of Poisson-Nernst-Planck (PNP) equations, a model of ion transport, plays a crucial role in the study of many physical and biological phenomena (cf. $[2,3,6,7,10,12,14,15,30,32,40,41,42,45,48,52,53,59,60]$ ). However, when ions are crowded in a narrow channel, the PNP equations become unreliable because the ion-size effect becomes important, but the PNP equations represent ions as point particles without size (cf. [1, 4, 8, 11, 18, 21, 22, 26, 33, 37, 38, $50,51,56,58,61]$ ). Hence the PNP equations need to be modified in order to describe solutions where the ion-size effect is important. Biological solutions are mixtures containing divalents in which ion size effects are always important (cf. [17, 19, 20]).

To modify the PNP equations, many efforts have been made to combine the energy functional of the PNP equations with the other exclusion terms which may come from liquid state theory and density functional theory [5, 23, 34, 38, 40, 47]. Being related to liquid state theory, the Lennard-Jones (LJ) potential is often used as an approximate model of the van der Waals force (cf. [29, 46]). However, it seems that no one had ever used the LJ potential to modify the PNP equations before the pioneering works of Eisenberg and Liu, who derived the PNP equations with size effects, i.e. equations (2.6)-(2.8), by combining the repulsive term of the LJ potential and the energy functional of the PNP equations (cf. [16, 28]).

Equations (2.6)-(2.8) importantly generalize the PNP equations and numerically simulate the selectivity of ion channels which can not be obtained by solving the PNP equations alone (cf. [62, 63, 64, 65, 66]). Nevertheless, due to imposing the LJ potential, equations (2.6)-(2.8) become a complicated system of differential-integral equations having no numerical efficiency (cf. [17, 28]) and allowing no theoretical result. The goal of this paper is to approximate the LJ potential and simplify equations (2.6)-(2.8) into the PNP-steric equations, a new mathematical model for the LJ interaction in ionic solutions. Instead of singular integrals of equations (2.6)-(2.8), the PNP-steric equations are composed of the PNP equations and nonlinear differential terms with coupling constants. Numerical simulations of the PNP-steric equations are presented in [25], which shows the numerical efficiency to simulate the selectivity of ion channels previously studied in Monte Carlo simulations with results comparable to a wide range of experiments.

The main difficulty of numerical simulation of the PNP-steric equations is how to choose coupling constants suitably. This motivates us to study a simple case (one anion and one cation species) of the PNP-steric equations with the Dirichlet boundary conditions using suitable asymptotic expansions to see the effect of coupling constants $g_{i j}$ 's. The formal asymptotic analysis gives the stability and instability conditions represented by the $g_{i j}$ 's which can be regarded as the first step in a series of analyses. (see sections 4 and 5). More theoretical results will be done soon which may be useful for the choice of coupling constants in order to do further numerical simulations on ion channels.

This paper has two major parts: one is the approximation of the LJ potential 
using band-limited functions and the other is the stability and instability conditions for the PNP-steric equations with the Dirichlet boundary conditions using asymptotic expansions. The approximate LJ potentials are introduced in Section 2.1 and the detailed mathematical arguments are stated in Section 3. The PNP-steric equations are derived in Section 2.2 and the stability and instability conditions for the PNPsteric equations are proved in Section 4 and 5, respectively.

\section{Preliminaries}

2.1. The approach to the Lennard-Jones potential. Because the LJ potential $\Psi$ does not have a Fourier transform, it is difficult to study the energy functional $E_{L J}$ directly. When the spatial frequency variable $\xi$ is bounded, the Fourier transform $\widehat{\Psi \chi_{\sigma}}(\xi)$ tends to infinity as $\sigma$ goes to zero, but by the Riemann-Lebesgue Lemma, $\lim _{|\xi| \rightarrow \infty} \widehat{\Psi \chi_{\sigma}}(\xi)=0$ for all $\sigma>0$. Hence the asymptotic behavior of the Fourier transform $\widehat{\Psi \chi_{\sigma}}(\xi)$ is dominated at bounded spatial frequencies and negligible at high spatial frequencies. This gives a reason to cut off high spatial frequencies in order to see the asymptotic behavior of the Fourier transform $\widehat{\Psi \chi_{\sigma}}(\xi)$. From a physical and biological point of view, it seems obvious that particularly small spatial features cannot be used by evolution to produce biological function and so should be absent in analysis. The high spatial frequency cut-off function $\varphi_{\sigma}$ is a band-limited function defined by

$$
\varphi_{\sigma}(x)=\left(1-\chi_{\sigma^{-\gamma}}(\xi)\right)^{v},
$$

for $x, \xi \in \mathbb{R}^{d}$, where $v$ denotes the inverse Fourier transform. Obviously,

$$
\hat{\varphi}_{\sigma}(\xi)=1-\chi_{\sigma^{-\gamma}}(\xi)=\left\{\begin{array}{rrr}
1, & \text { if } & |\xi| \leq \sigma^{-\gamma} \\
0, & \text { if } & |\xi|>\sigma^{-\gamma}
\end{array}\right.
$$

for $\xi \in \mathbb{R}^{d}$, where the hat denotes the Fourier transform, and $0<\gamma<1$ is a constant independent of $\sigma$ and $\xi$.

Generically, band-limited functions can handle spatial information locally in frequency domains and play important roles in Fourier analysis of mathematics and have many applications to engineering, physics, and statistics (cf. [31, 35, 55]). Physically, it is obvious that the band-limited approximation is reasonable if not inevitable. After all, derivations of PNP replace Maxwell's equations with Poisson's equation, and neglect 'capacitance to ground' (capacitive coupling between ionic solutions and nearby ground planes) always present in experiments. These and many other effects at high frequencies are better attenuated to zero than approximated irrationally (i.e., without known error bounds) as they are in treatments with unlimited bandwidth. Here a new approach to the LJ potential $\Psi$ uses the spatially band-limited function $\varphi_{\sigma}$ to define the approximate potential $\Psi$ as follows:

$$
\Psi_{\sigma}(z)=\left(\Psi \chi_{\sigma}\right) \star \varphi_{\sigma}(z) \quad \text { for } \quad z \in \mathbb{R}^{d},
$$

where the asterisk is the standard convolution, $\varphi_{\sigma}$ is the spatially band-limited function defined in (2.1), and $\chi_{\sigma}$ is the characteristic function of the exterior ball $\left\{z \in \mathbb{R}^{d}:|z|>\sigma\right\}$. Note that $\chi_{\sigma}(z)$ and $1-\chi_{\sigma^{-\gamma}}(\xi)$ are the characteristic functions of $\left\{z \in \mathbb{R}^{d}:|z|>\sigma\right\}$ and $\left\{\xi \in \mathbb{R}^{d}:|\xi| \leq \sigma^{-\gamma}\right\}$, respectively, and both of them extend to the entire space $\mathbb{R}^{d}$ as the small parameter $\sigma$ goes to zero. As $\sigma$ goes to zero, $\chi_{\sigma} \sim 1$, $1-\chi_{\sigma^{-\gamma}} \sim 1$, and

$$
\widehat{\Psi_{\sigma}}(\xi)=\widehat{\Psi \chi_{\sigma}}(\xi) \widehat{\varphi_{\sigma}}(\xi)=\widehat{\Psi \chi_{\sigma}}(\xi)\left[1-\chi_{\sigma^{-\gamma}}(\xi)\right] \sim \widehat{\Psi}(\xi) .
$$


which implies $\Psi_{\sigma} \sim \Psi$. Hence formally,

$$
\int_{\mathbb{R}^{d}} c_{i}(x)\left(\Psi_{\sigma} \star c_{j}\right)(x) d x \sim \int_{\mathbb{R}^{d}} c_{i}(x)\left(\Psi \star c_{j}\right)(x) d x=E_{L J}\left[c_{i}, c_{j}\right] .
$$

This shows how we approximate the energy functional $E_{L J}$.

The approximate energy functional $E_{L J, \sigma}$ is defined by

$$
E_{L J, \sigma}\left[c_{i}, c_{j}\right]=\iint_{\mathbb{R}^{d} \times \mathbb{R}^{d}} \Psi_{\sigma}(x-y) c_{i}(x) c_{j}(y) d x d y=\int_{\mathbb{R}^{d}} c_{i}(x)\left(\Psi_{\sigma} \star c_{j}\right)(x) d x .
$$

As $\sigma$ goes to zero, the functional $E_{L J, \sigma}$ tends to the functional $E_{L J}$ if the following hypothesis holds:

$$
\lim _{\sigma \rightarrow 0+}\left[\int_{\mathbb{R}^{d}} \widehat{\Psi \chi_{\sigma}}(\xi)\left[1-\chi_{\sigma^{-\gamma}}(\xi)\right] \widehat{c_{i}}(\xi) \widehat{c_{j}}(\xi) d \xi-\int_{\mathbb{R}^{d}} \widehat{\Psi \chi_{\sigma}}(\xi) \widehat{c_{i}}(\xi) \widehat{c_{j}}(\xi) d \xi\right]=0
$$

(see Proposition 3.1). Here the meaning of 'approximate' is different from that of conventional approximation theory. Note that the characteristic function $1-\chi_{\sigma^{-\gamma}}$ truncates the high frequencies $|\xi|>\sigma^{-\gamma}$, but still preserves the spatial frequencies of order $|\xi| \sim \sigma^{-\theta}$ (for all $0<\theta<\gamma$ ) tending to infinity as $\sigma$ goes to zero. By standard theorems of Fourier analysis (cf. [54]), the functional $E_{L J, \sigma}$ satisfies $E_{L J, \sigma}\left[c_{i}, c_{j}\right]=$ $\int_{\mathbb{R}^{d}} \widehat{c_{i}}(\xi) \widehat{\Psi_{\sigma} \star c_{j}}(\xi) d \xi=\int_{\mathbb{R}^{d}} \widehat{c_{i}}(\xi) \widehat{\Psi_{\sigma}}(\xi) \widehat{c_{j}}(\xi) d \xi$ On the other hand, (2.2) and (2.3) imply $\widehat{\Psi_{\sigma}}(\xi) \sim C_{1} \frac{\omega_{d}}{12-d} \sigma^{d-12}$ as $\sigma$ goes to zero, where $\omega_{d}$ is the surface area of $d$ dimensional unit ball (see the proof of Proposition 3.2). Hence

$$
E_{L J, \sigma}\left[c_{i}, c_{j}\right] \sim C_{1} \frac{\omega_{d}}{12-d} \sigma^{d-12} \int_{\mathbb{R}^{d}} \widehat{c_{i}}(\xi) \widehat{c_{j}}(\xi) d \xi=C_{1} \frac{\omega_{d}}{12-d} \sigma^{d-12} \int_{\mathbb{R}^{d}} c_{i}(x) c_{j}(x) d x .
$$

This shows that the functional $E_{L J, \sigma}$ is asymptotically close to another functional $\tilde{E}_{L J, \sigma}$ as follows:

$$
E_{L J, \sigma}\left[c_{i}, c_{j}\right] \sim \tilde{E}_{L J, \sigma}\left[c_{i}, c_{j}\right]=C_{1} S_{\sigma} \int_{\mathbb{R}^{d}} c_{i}(x) c_{j}(x) d x
$$

where $S_{\sigma}=\frac{\omega_{d}}{12-d} \sigma^{d-12}$. Thus the functional $\tilde{E}_{L J, \sigma}$ can be regarded as an approximate energy functional to $E_{L J}$. Note that the constant $C_{1}$ comes from the repulsive term of the LJ potential, but the constant $C_{2}$ (for the attractive term of the LJ potential) does not affect the leading term of the asymptotic behavior of $\widehat{\Psi_{\sigma}}$. This may support the work of $[16,17,18,28]$ which only use the repulsive term of the LJ potential to describe ionic interactions. However, the effects of the attractive terms remain to be investigated. They may have been selected by evolution to produce qualitative behavior of importance to biology. Note that the functional $\tilde{E}_{L J, \sigma}$ is much simpler than the functional $E_{L J}$ and it can be expressed as

$$
\tilde{E}_{L J, \sigma}\left[c_{i}, c_{j}\right]=\iint_{\mathbb{R}^{d} \times \mathbb{R}^{d}} C_{1} S_{\sigma} \delta_{0}(x-y) c_{i}(x) c_{j}(y) d x d y,
$$

being the same as replacing the LJ potential $\Psi$ by a delta function $C_{1} S_{\sigma} \delta_{0}$ in the energy functional $E_{L J}$, where $\delta_{0}(\cdot)$ is the standard delta function concentrated at the origin. Such a simple energy functional $\tilde{E}_{L J, \sigma}$ can be used to derive the PNP-steric equations as a new model of ionic solutions. 
2.2. PNP-steric equations. The Poisson-Nernst-Planck (PNP) equations consist of the Nernst-Planck equations coupled with the Poisson equation being expressed as follows:

$$
\left\{\begin{array}{l}
\frac{\partial c_{i}}{\partial t}=\frac{D_{i}}{k_{B} T} \nabla \cdot\left(c_{i} \nabla \frac{\delta E_{p n p}}{\delta c_{i}}\right), \quad i=1, \cdots, N \\
-\nabla \cdot(\varepsilon \nabla \phi)=\rho_{0}+\sum_{i=1}^{N} z_{i} e c_{i},
\end{array}\right.
$$

where $E_{p n p}$ is the energy functional of the PNP equations given by

$$
E_{p n p}\left[c_{1}, \cdots, c_{N}, \phi\right]:=\int_{\mathbb{R}^{d}}\left[k_{B} T \sum_{i=1}^{N} c_{i} \log c_{i}+\frac{1}{2}\left(\rho_{0}+\sum_{i=1}^{N} z_{i} e c_{i}\right) \phi\right] d x .
$$

Here $N$ is the number of ion species, $c_{i}$ is the distribution function, $D_{i}$ is the diffusion constant, and $z_{i}$ is the valence of the $i$ th ion species, respectively. Besides, $\phi$ is the electrostatic potential, $\varepsilon$ is the dielectric constant, $\rho_{0}$ is the permanent (fixed) charge density of the system, $k_{B}$ is the Boltzmann constant, $T$ is the absolute temperature, and $e$ is the elementary charge. More precisely, the PNP equations are denoted as

$$
\left\{\begin{array}{l}
\frac{\partial c_{i}}{\partial t}=D_{i} \nabla \cdot\left(\nabla c_{i}+\frac{z_{i} e}{k_{B} T} c_{i} \nabla \phi\right), \quad i=1, \cdots, N, \\
-\nabla \cdot(\varepsilon \nabla \phi)=\rho_{0}+\sum_{i=1}^{N} z_{i} e c_{i} .
\end{array}\right.
$$

To include the hard sphere repulsion of ions, the energy functional $E_{p n p}$ is modified by adding the energy functional $\sum_{i, j=1}^{N} E_{L J}\left[c_{i}, c_{j}\right]$ with the constants $C_{1}=$ $\frac{1}{2} \epsilon_{i j}\left(a_{i}+a_{j}\right)^{12}$ and $C_{2}=0$, where $c_{i}$ and $c_{j}$ are the distribution (concentration) functions of the $i$ th and $j$ th ion species with the radii $a_{i}$ and $a_{j}$, respectively. Then the modified energy functional $E_{m p n p}$ becomes

$$
\begin{aligned}
E_{m p n p}\left[c_{1}, \cdots, c_{N}, \phi\right]:= & \int_{\mathbb{R}^{d}}\left(k_{B} T \sum_{i=1}^{N} c_{i} \log c_{i}+\frac{1}{2}\left(\rho_{0}+\sum_{i=1}^{N} z_{i} e c_{i}\right) \phi\right) d x \\
& +\frac{1}{2} \sum_{i, j=1}^{N} \iint_{\mathbb{R}^{d} \times \mathbb{R}^{d}} \frac{\epsilon_{i j}\left(a_{i}+a_{j}\right)^{12}}{|x-y|^{12}} c_{i}(x) c_{j}(y) d x d y
\end{aligned}
$$

Using energy variational analysis (cf. $[16,28]$ ), the modified PNP equations are the equations

$$
\frac{\partial c_{i}}{\partial t}=\frac{D_{i}}{k_{B} T} \nabla \cdot\left(c_{i} \nabla \frac{\delta E_{m p n p}}{\delta c_{i}}\right), \quad i=1, \cdots, N
$$

i.e.

$$
\frac{\partial c_{i}}{\partial t}+\nabla \cdot J_{i}=0, \quad i=1, \cdots, N
$$


coupled with the Poisson equation

$$
-\nabla \cdot(\varepsilon \nabla \phi)=\rho_{0}+\sum_{i=1}^{N} z_{i} e c_{i},
$$

where the flux $J_{i}$ is

$$
J_{i}=-D_{i} \nabla c_{i}-\frac{D_{i} c_{i}}{k_{B} T} z_{i} e \nabla \phi-\frac{D_{i} c_{i}}{k_{B} T} \sum_{j=1}^{N} \nabla \int_{\mathbb{R}^{d}} \frac{\epsilon_{i j}\left(a_{i}+a_{j}\right)^{12}}{|x-y|^{12}} c_{j}(y) d y .
$$

However, equations (2.6)-(2.8) are difficult to investigate theoretically because they are partial differential-integral equations with singular integrals. Moreover, due to the effect of high (Fourier) frequencies, the numerical computations of equations (2.6)(2.8) may lose accuracy and become inefficient (cf. [28]).

Instead of equations (2.6)-(2.8), a simple model can be derived by replacing the energy functional $\sum_{i, j=1}^{N} E_{L J}\left[c_{i}, c_{j}\right]$ (with $C_{1}=\frac{1}{2} \epsilon_{i j}\left(a_{i}+a_{j}\right)^{12}$ and $C_{2}=0$ ) by the approximate energy functional

$$
\sum_{i, j=1}^{N} \tilde{E}_{L J, \sigma}\left[c_{i}, c_{j}\right]=\frac{1}{2} \epsilon_{i j}\left(a_{i}+a_{j}\right)^{12} S_{\sigma} \int_{\mathbb{R}^{d}} c_{i}(x) c_{j}(x) d x
$$

defined in (2.4). Then the energy functional $E_{m p n p}$ can be approximated by

$$
\begin{aligned}
E_{\sigma}\left[c_{1}, \cdots, c_{N}, \phi\right]:=\int_{\mathbb{R}^{d}} & \left(k_{B} T \sum_{i=1}^{N} c_{i} \log c_{i}+\frac{1}{2}\left(\rho_{0}+\sum_{i=1}^{N} z_{i} e c_{i}\right) \phi\right) d x \\
& +\frac{1}{2} \sum_{i, j=1}^{N} \epsilon_{i j}\left(a_{i}+a_{j}\right)^{12} S_{\sigma} \int_{\mathbb{R}^{d}} c_{i}(x) c_{j}(x) d x
\end{aligned}
$$

which is much simpler than the energy functional $E_{m p n p}$. Using energy variational analysis (cf. $[16,28]$ ), a new model called the PNP-steric equations is expressed by

$$
\frac{\partial c_{i}}{\partial t}=\frac{D_{i}}{k_{B} T} \nabla \cdot\left(c_{i} \nabla \frac{\delta E_{\sigma}}{\delta c_{i}}\right), \quad i=1, \cdots, N
$$

i.e.

$$
\frac{\partial c_{i}}{\partial t}+\nabla \cdot J_{i}=0
$$

coupled with the Poisson equation

$$
-\nabla \cdot(\varepsilon \nabla \phi)=\rho_{0}+\sum_{i=1}^{N} z_{i} e c_{i}
$$

where the flux $J_{i}$ is

$$
J_{i}=-D_{i} \nabla c_{i}-\frac{D_{i} c_{i}}{k_{B} T} z_{i} e \nabla \phi-\frac{D_{i} c_{i}}{k_{B} T} S_{\sigma} \sum_{j=1}^{N} \epsilon_{i j}\left(a_{i}+a_{j}\right)^{12} \nabla c_{j} .
$$


Here the symmetry $\epsilon_{i j}=\epsilon_{j i}$ has been assumed for notation convenience. The PNPsteric equations are of convection-diffusion type with the following energy dissipation law:

$$
\frac{d}{d t} E_{\sigma}\left[c_{1}, \cdots, c_{N}, \phi\right]=-\int_{\mathbb{R}^{d}} \sum_{i=1}^{N} \frac{D_{i} c_{i}}{k_{B} T}\left|\nabla\left(k_{B} T \log c_{i}+z_{i} e \phi+\mu_{i}\right)\right|^{2} d x
$$

where $\mu_{i}=\frac{\delta}{\delta c_{i}} \sum_{j, k=1}^{N} \tilde{E}_{L J, \sigma}\left[c_{j}, c_{k}\right]=S_{\sigma} \sum_{j=1}^{N} \epsilon_{i j}\left(a_{i}+a_{j}\right)^{12} c_{j}$ is the chemical potential. On the other hand, the PNP-steric equations have more nonlinear differential terms than the PNP equations so they can simulate the selectivity of ion channels efficiently (cf. [25]). Note that the selectivity of ion channels can not be found by using the PNP equations. This shows that the PNP-steric equations are more capable than the PNP equations and much simpler than equations (2.6)-(2.8).

For the case of two species ions (i.e. $N=2$ ) with one anionic and cationic species, the index $j=1,2$ is replaced by $j=n, p$ for notation convenience and the PNP-steric equations (2.9)-(2.11) are presented as

$$
\begin{aligned}
\frac{\partial c_{n}}{\partial t} & =D_{n}\left[\nabla \cdot\left(\nabla c_{n}+\frac{z_{n} e}{k_{B} T} c_{n} \nabla \phi\right)+\tilde{S}_{\sigma} \nabla \cdot\left(g_{n n} c_{n} \nabla c_{n}+g_{n p} c_{n} \nabla c_{p}\right)\right], \\
\frac{\partial c_{p}}{\partial t} & =D_{p}\left[\nabla \cdot\left(\nabla c_{p}+\frac{z_{p} e}{k_{B} T} c_{p} \nabla \phi\right)+\tilde{S}_{\sigma} \nabla \cdot\left(g_{p p} c_{p} \nabla c_{p}+g_{n p} c_{p} \nabla c_{n}\right)\right], \\
-\nabla \cdot(\varepsilon \nabla \phi) & =\rho_{0}+z_{n} e c_{n}+z_{p} e c_{p},
\end{aligned}
$$

for $x \in \mathbb{R}^{d}, t>0$, where the function $c_{n}$ is for anion concentration, $c_{p}$ is for cation concentration, $\tilde{S}_{\sigma}=\frac{1}{k_{B} T} S_{\sigma}, g_{n p}=\epsilon_{12}\left(a_{1}+a_{2}\right)^{12}, g_{n n}=\epsilon_{11}\left(2 a_{1}\right)^{12}$, and $g_{p p}=\epsilon_{22}\left(2 a_{2}\right)^{12}$. Due to the spatial dimension $d \leq 3$, the constant $\tilde{S}_{\sigma} \sim \sigma^{d-12}$ becomes a large quantity tending to infinity as $\sigma$ goes to zero. Let $\tilde{\varepsilon}=\frac{k_{B} T}{e^{2}} \varepsilon, \tilde{\delta}=1 / \tilde{S}_{\sigma}, \tau=t / \tilde{\delta}, \tilde{\phi}(x, \tau)=$ $\frac{e}{k_{B} T} \phi(x, t)$, and $\tilde{c}_{j}(x, \tau)=c_{j}(x, t)$ for $j=n, p$. Then the equations (2.12)-(2.14) are transformed into

$$
\begin{aligned}
\frac{1}{D_{n}} \frac{\partial \tilde{c}_{n}}{\partial \tau} & =\tilde{\delta} \nabla \cdot\left(\nabla \tilde{c}_{n}+z_{n} \tilde{c}_{n} \nabla \tilde{\phi}\right)+\nabla \cdot\left(g_{n n} \tilde{c}_{n} \nabla \tilde{c}_{n}+g_{n p} \tilde{c}_{n} \nabla \tilde{c}_{p}\right), \\
\frac{1}{D_{p}} \frac{\partial \tilde{c}_{p}}{\partial \tau} & =\tilde{\delta} \nabla \cdot\left(\nabla \tilde{c}_{p}+z_{p} \tilde{c}_{p} \nabla \tilde{\phi}\right)+\nabla \cdot\left(g_{p p} \tilde{c}_{p} \nabla \tilde{c}_{p}+g_{n p} \tilde{c}_{p} \nabla \tilde{c}_{n}\right), \\
-\nabla \cdot(\tilde{\varepsilon} \nabla \tilde{\phi}) & =\tilde{\rho}_{0}+z_{n} \tilde{c}_{n}+z_{p} \tilde{c}_{p},
\end{aligned}
$$

for $x \in \mathbb{R}^{d}, \tau>0$, where $\tilde{\rho}_{0}=\frac{\rho_{0}}{e}$. Note that $\frac{\partial c_{j}}{\partial t}=\tilde{\delta}^{-1} \frac{\partial \tilde{c}_{j}}{\partial \tau}$ for $j=n, p$, and $\tau \sim 1$ is equivalent to $t \sim \tilde{\delta}=1 / \tilde{S}_{\sigma}$, being a small time scale. For notation convenience, removing the tilde and replacing $\tau$ by $t$, the equations (2.15)-(2.17) become

$$
\begin{aligned}
\frac{1}{D_{n}} \frac{\partial c_{n}}{\partial t} & =\delta \nabla \cdot\left(\nabla c_{n}+z_{n} c_{n} \nabla \phi\right)+\nabla \cdot\left(g_{n n} c_{n} \nabla c_{n}+g_{n p} c_{n} \nabla c_{p}\right), \\
\frac{1}{D_{p}} \frac{\partial c_{p}}{\partial t} & =\delta \nabla \cdot\left(\nabla c_{p}+z_{p} c_{p} \nabla \phi\right)+\nabla \cdot\left(g_{p p} c_{p} \nabla c_{p}+g_{n p} c_{p} \nabla c_{n}\right), \\
-\nabla \cdot(\varepsilon \nabla \phi) & =\rho_{0}+z_{n} c_{n}+z_{p} c_{p},
\end{aligned}
$$

for $x \in \mathbb{R}^{d}, t>0$. 
Instead of the entire space $\mathbb{R}^{d}$, here the spatial domain is considered as an onedimensional $(d=1)$ interval $(-1,1)$, and then the equations $(2.18)-(2.20)$ are changed into the following equations:

$$
\begin{array}{rlrl}
\frac{1}{D_{n}} \frac{\partial c_{n}}{\partial t} & =\delta \frac{\partial}{\partial x} \cdot\left(\frac{\partial c_{n}}{\partial x}+z_{n} c_{n} \frac{\partial \phi}{\partial x}\right)+\frac{\partial}{\partial x}\left(g_{n n} c_{n} \frac{\partial c_{n}}{\partial x}+g_{n p} c_{n} \frac{\partial c_{p}}{\partial x}\right), \text { for } x \in(-1,1), t>0 \\
\frac{1}{D_{p}} \frac{\partial c_{p}}{\partial t} & =\delta \frac{\partial}{\partial x}\left(\frac{\partial c_{p}}{\partial x}+z_{p} c_{p} \frac{\partial \phi}{\partial x}\right)+\frac{\partial}{\partial x}\left(g_{p p} c_{p} \frac{\partial c_{p}}{\partial x}+g_{n p} c_{p} \frac{\partial c_{n}}{\partial x}\right), & \text { for } x \in(-1,1), t>0 \\
-\varepsilon \frac{\partial^{2} \phi}{\partial x^{2}} & =\rho_{0}+z_{n} c_{n}+z_{p} c_{p}, & & \text { for } x \in(-1,1), t>0 .
\end{array}
$$

Setting $\varepsilon=1$ and $\rho_{0}=0$, these equations become

$$
\begin{array}{rlrl}
\frac{1}{D_{n}} \frac{\partial c_{n}}{\partial t} & =\delta \frac{\partial}{\partial x}\left(\frac{\partial c_{n}}{\partial x}+z_{n} c_{n} \frac{\partial \phi}{\partial x}\right)+\frac{\partial}{\partial x}\left(g_{n n} c_{n} \frac{\partial c_{n}}{\partial x}+g_{n p} c_{n} \frac{\partial c_{p}}{\partial x}\right), \text { for } x \in(-1,1), t>0 \\
\frac{1}{D_{p}} \frac{\partial c_{p}}{\partial t} & =\delta \frac{\partial}{\partial x}\left(\frac{\partial c_{p}}{\partial x}+z_{p} c_{p} \frac{\partial \phi}{\partial x}\right)+\frac{\partial}{\partial x}\left(g_{p p} c_{p} \frac{\partial c_{p}}{\partial x}+g_{n p} c_{p} \frac{\partial c_{n}}{\partial x}\right), \text { for } x \in(-1,1), t>0 \\
-\frac{\partial^{2} \phi}{\partial x^{2}} & =z_{n} c_{n}+z_{p} c_{p}, & \text { for } x \in(-1,1), t>0,
\end{array}
$$

where $\delta$ is a small parameter tending to zero. For simplicity, we consider the following Dirichlet boundary conditions:

$$
\left\{\begin{array}{l}
c_{n}=-\frac{B_{1}}{z_{n}}, c_{p}=\frac{B_{1}}{z_{p}}, \phi=B_{3}, \text { as } x=1, \\
c_{n}=-\frac{B_{2}}{z_{n}}, c_{p}=\frac{B_{2}}{z_{p}}, \phi=B_{4}, \text { as } x=-1,
\end{array}\right.
$$

where $B_{k}, k=1, \cdots, 4$ are constants. Here we only focus on the equations (2.21)-(2.23) with the boundary condition (2.24), but not (2.18)-(2.20) on the entire space. Using the formal asymptotic analysis, we may get the stability and instability conditions of the equations (2.21)-(2.23) with the boundary condition (2.24), which show the effect of coupling constants $g_{i j}$ 's.

To see the solution of the equations (2.21)-(2.23), the following asymptotic expansions are used:

$$
\begin{aligned}
& c_{n}=c_{n, 0}+\delta c_{n, 1}+\delta^{2} c_{n, 2}+\cdots, \\
& c_{p}=c_{p, 0}+\delta c_{p, 1}+\delta^{2} c_{p, 2}+\cdots, \\
& \phi=\phi_{0}+\delta \phi_{1}+\delta^{2} \phi_{2}+\cdots
\end{aligned}
$$

Then the zeroth order solution $\left(c_{n, 0}, c_{p, 0}, \phi_{0}\right)$ satisfies

$$
\begin{gathered}
\frac{1}{D_{n}} \frac{\partial c_{n, 0}}{\partial t}=\frac{\partial}{\partial x}\left(g_{n n} c_{n, 0} \frac{\partial c_{n, 0}}{\partial x}+g_{n p} c_{n, 0} \frac{\partial c_{p, 0}}{\partial x}\right), \\
\frac{1}{D_{p}} \frac{\partial c_{p, 0}}{\partial t}=\frac{\partial}{\partial x}\left(g_{p p} c_{p, 0} \frac{\partial c_{p, 0}}{\partial x}+g_{n p} c_{p, 0} \frac{\partial c_{n, 0}}{\partial x}\right),
\end{gathered}
$$




$$
-\frac{\partial^{2} \phi_{0}}{\partial x^{2}}=z_{n} c_{n, 0}+z_{p} c_{p, 0}
$$

for $x \in(-1,1)$ and $t>0$. As $g_{n p}=0$, both $(2.25)$ and (2.26) become the standard porous medium equation (cf. [57]); hence for $g_{n p} \neq 0$, the equations (2.25) and (2.26) can be regarded as a coupled system of porous medium equations.

The steady state equations of (2.25) and (2.26) are denoted as

$$
\begin{aligned}
\left(g_{n n} c_{n, 0} c_{n, 0}^{\prime}+g_{n p} c_{n, 0} c_{p, 0}^{\prime}\right)^{\prime}(x) & =0, \\
\left(g_{p p} c_{p, 0} c_{p, 0}^{\prime}+g_{n p} c_{p, 0} c_{n, 0}^{\prime}\right)^{\prime}(x) & =0,
\end{aligned}
$$

for $x \in(-1,1)$, where a prime mark ( $I)$ denotes differentiation of the spatial variable $x$. Electroneutrality (which means the charge of anions is equal to that of cations) holds in most biological systems (cf. [67]). A particular assumption called pointwise electroneutrality, which means the charge of anions is everywhere equal to that of cations, appears in the zeroth order equation [24] as a pleasingly natural physical approximation. To find solutions of (2.28)-(2.29) with pointwise electroneutrality, it is natural to assume that $c_{n, 0}$ and $c_{p, 0}$ have the form

$$
c_{n, 0}(x)=z_{p} w(x), \quad c_{p, 0}(x)=-z_{n} w(x) \quad \text { for } \quad x \in(-1,1),
$$

so the total charge of $c_{n, 0}$ and $c_{p, 0}$ becomes zero, i.e. $z_{n} c_{n, 0}(x)+z_{p} c_{p, 0}(x)=0$ for $x \in$ $(-1,1)$. Note that $z_{n}$ and $z_{p}$ are the associated valences, the charge on an individual ion. To solve the equations (2.28) and (2.29), the function $w=w(x)$ must satisfy $\left(w^{2}\right)^{\prime \prime}(x)=0$ for $x \in(-1,1)$, and hence $w=w(x)=\sqrt{K_{1} x+K_{0}}$, where $K_{0}$ and $K_{1}$ are constants determined by the Dirichlet boundary conditions as follows:

$$
-z_{n} c_{n, 0}(1)=z_{p} c_{p, 0}(1)=B_{1}, \quad-z_{n} c_{n, 0}(-1)=z_{p} c_{p, 0}(-1)=B_{2},
$$

and $B_{1}$ and $B_{2}$ are the positive constants for the Dirichlet boundary conditions in (2.24). Because $w(x)=\sqrt{K_{1} x+K_{0}}$ for $x \in[-1,1]$, by $(2.30)$ and (2.31), it is obvious that $\sqrt{K_{1}+K_{0}}=w(1)=\frac{B_{1}}{-z_{n} z_{p}}$ and $\sqrt{K_{0}-K_{1}}=w(-1)=\frac{B_{2}}{-z_{n} z_{p}}$, which imply

$$
K_{1}=\frac{B_{1}^{2}-B_{2}^{2}}{2 z_{n}^{2} z_{p}^{2}} \quad \text { and } \quad K_{0}=\frac{B_{1}^{2}+B_{2}^{2}}{2 z_{n}^{2} z_{p}^{2}} .
$$

Hence

$$
w(x)=\frac{\sqrt{\left(B_{1}^{2}-B_{2}^{2}\right) x+B_{1}^{2}+B_{2}^{2}}}{\sqrt{2}\left|z_{n} z_{p}\right|}>0 \quad \text { for } \quad x \in[-1,1],
$$

being non-constant if $B_{1} \neq B_{2}$. Combining (2.27) and (2.30), the electric potential $\phi_{0}$ satisfies the following equation:

$$
-\phi_{0}^{\prime \prime}(x)=0 \quad \text { for } \quad x \in(-1,1) .
$$

The equation (2.33) is a standard differential equation solved as follows:

$$
\phi_{0}(x)=a_{0} x+b_{0} \quad \text { for } \quad x \in(-1,1),
$$

where $a_{0}$ and $b_{0}$ are constants determined by the boundary conditions of $\phi_{0}$ given in (2.24). The first order solution $\left(c_{n, 1}, c_{p, 1}, \phi_{1}\right)=\left(c_{n, 1}(x, t), c_{p, 1}(x, t), \phi_{1}(x, t)\right)$ satisfies

$$
\frac{1}{D_{n}} \frac{\partial c_{n, 1}}{\partial t}=\frac{\partial}{\partial x}\left(\frac{\partial c_{n, 0}}{\partial x}+z_{n} c_{n, 0} \frac{\partial \phi_{0}}{\partial x}\right)+\frac{\partial}{\partial x}\left[g_{n n} \frac{\partial\left(c_{n, 0} c_{n, 1}\right)}{\partial x}+g_{n p}\left(\frac{\partial c_{p, 0}}{\partial x} c_{n, 1}+c_{n, 0} \frac{\partial c_{p, 1}}{\partial x}\right)\right],
$$




$$
\begin{aligned}
\frac{1}{D_{p}} \frac{\partial c_{p, 1}}{\partial t} & =\frac{\partial}{\partial x}\left(\frac{\partial c_{p, 0}}{\partial x}+z_{p} c_{p, 0} \frac{\partial \phi_{0}}{\partial x}\right)+\frac{\partial}{\partial x}\left[g_{p p} \frac{\partial\left(c_{p, 0} c_{p, 1}\right)}{\partial x}+g_{n p}\left(\frac{\partial c_{n, 0}}{\partial x} c_{p, 1}+c_{p, 0} \frac{\partial c_{n, 1}}{\partial x}\right)\right], \\
-\frac{\partial^{2} \phi_{1}}{\partial x^{2}} & =z_{n} c_{n, 1}+z_{p} c_{p, 1},
\end{aligned}
$$

for $x \in(-1,1), t>0$. By (2.30), the equations (2.35) and (2.36) become

$$
\begin{aligned}
& \frac{1}{D_{n}} \frac{\partial c_{n, 1}}{\partial t}=z_{p}\left(w^{\prime}+z_{n} w \phi_{0}^{\prime}\right)^{\prime}+\frac{\partial}{\partial x}\left[g_{n n} z_{p} \frac{\partial\left(w c_{n, 1}\right)}{\partial x}+g_{n p}\left(-z_{n} w^{\prime} c_{n, 1}+z_{p} w \frac{\partial c_{p, 1}}{\partial x}\right)\right] \\
& \frac{1}{D_{p}} \frac{\partial c_{p, 1}}{\partial t}=-z_{n}\left(w^{\prime}+z_{p} w \phi_{0}^{\prime}\right)^{\prime}+\frac{\partial}{\partial x}\left[-z_{n} g_{p p} \frac{\partial\left(w c_{p, 1}\right)}{\partial x}+g_{n p}\left(z_{p} w^{\prime} c_{p, 1}-z_{n} w \frac{\partial c_{n, 1}}{\partial x}\right)\right]
\end{aligned}
$$

for $x \in(-1,1), t>0$.

Let $\psi=z_{n} c_{n, 1}+z_{p} c_{p, 1}$ and $\varphi=-z_{n} c_{n, 1}+z_{p} c_{p, 1}$, i.e. $c_{n, 1}=\frac{1}{2 z_{n}}(\psi-\varphi)$ and $c_{p, 1}=$ $\frac{1}{2 z_{p}}(\psi+\varphi)$. Then after some algebraic calculations, the equations (2.38) and (2.39) can be transformed into the equations for $\psi$ and $\varphi$ as follows:

$$
\begin{aligned}
\frac{\partial \psi}{\partial t}=( & \left.D_{n}-D_{p}\right) z_{n} z_{p} w^{\prime \prime}(x)+z_{n} z_{p}\left(D_{n} z_{n}-D_{p} z_{p}\right)\left[w(x) \phi_{0}^{\prime}(x)\right]^{\prime} \\
& +\left(\tilde{d}_{n p}-\tilde{d}\right) \frac{\partial^{2}[w(x) \varphi]}{\partial x^{2}}+\left(\bar{g}+\bar{g}_{n p}\right) \frac{\partial}{\partial x}\left[w^{\prime}(x) \psi\right]+\left(\bar{g}-\bar{g}_{n p}\right) \frac{\partial}{\partial x}\left[w(x) \frac{\partial \psi}{\partial x}\right],
\end{aligned}
$$

and

$$
\begin{aligned}
\frac{\partial \varphi}{\partial t}=- & \left(D_{n}+D_{p}\right) z_{n} z_{p} w^{\prime \prime}(x)-z_{n} z_{p}\left(D_{n} z_{n}+D_{p} z_{p}\right)\left[w(x) \phi_{0}^{\prime}(x)\right]^{\prime} \\
& +\left(\bar{g}_{n p}+\bar{g}\right) \frac{\partial^{2}[w(x) \varphi]}{\partial x^{2}}+\left(\tilde{d}_{n p}-\tilde{d}\right) \frac{\partial}{\partial x}\left[w^{\prime}(x) \psi\right]-\left(\tilde{d}_{n p}+\tilde{d}\right) \frac{\partial}{\partial x}\left[w(x) \frac{\partial \psi}{\partial x}\right],
\end{aligned}
$$

where $\bar{g}_{n p}, \tilde{d}_{n p}, \bar{g}$, and $\tilde{d}$ are constants defined as follows:

$$
\left\{\begin{aligned}
\bar{g}_{n p} & =\frac{1}{2} g_{n p}\left(D_{p} z_{p}-D_{n} z_{n}\right), \\
\tilde{d}_{n p} & =\frac{1}{2} g_{n p}\left(D_{p} z_{p}+D_{n} z_{n}\right), \\
\bar{g} & =\frac{1}{2}\left(D_{n} z_{p} g_{n n}-D_{p} z_{n} g_{p p}\right), \\
\tilde{d} & =\frac{1}{2}\left(D_{n} z_{p} g_{n n}+D_{p} z_{n} g_{p p}\right) .
\end{aligned}\right.
$$

The equations (2.40) and (2.41) depend on valences $z_{i}$, diffusion constants $D_{i}$, and coefficients $g_{i j} \sim\left(a_{i}+a_{j}\right)^{12}(i, j=n, p)$ related to ionic radii. In particular, these equations can represent the difference between $\mathrm{NaCl}, \mathrm{KCl}$, and $\mathrm{CaCl}_{2}$.

For notational convenience, the equations (2.40) and (2.41) can be expressed as

$$
\frac{\partial}{\partial t}\left(\begin{array}{c}
\psi \\
\varphi
\end{array}\right)=\left(\begin{array}{l}
f_{1} \\
f_{2}
\end{array}\right)+\left(\begin{array}{ll}
L_{11} & L_{12} \\
L_{21} & L_{22}
\end{array}\right)\left(\begin{array}{c}
\psi \\
\varphi
\end{array}\right)
$$

where $f_{1}$ and $f_{2}$ are external force functions given by

$$
f_{1}(x)=\left(D_{n}-D_{p}\right) z_{n} z_{p} w^{\prime \prime}(x)+z_{n} z_{p}\left(D_{n} z_{n}-D_{p} z_{p}\right)\left[w(x) \phi_{0}^{\prime}(x)\right]^{\prime},
$$




$$
f_{2}(x)=-\left(D_{n}+D_{p}\right) z_{n} z_{p} w^{\prime \prime}(x)-z_{n} z_{p}\left(D_{n} z_{n}+D_{p} z_{p}\right)\left[w(x) \phi_{0}^{\prime}(x)\right]^{\prime},
$$

and $L_{i j}, i, j=1,2$ are differential operators defined by

$$
\begin{aligned}
& L_{11} \psi=\left(\bar{g}+\bar{g}_{n p}\right) \frac{\partial}{\partial x}\left[w^{\prime}(x) \psi\right]+\left(\bar{g}-\bar{g}_{n p}\right) \frac{\partial}{\partial x}\left[w(x) \frac{\partial \psi}{\partial x}\right], \\
& L_{12} \varphi=\left(\tilde{d}_{n p}-\tilde{d}\right) \frac{\partial^{2}[w(x) \varphi]}{\partial x^{2}}, \\
& L_{21} \psi=\left(\tilde{d}_{n p}-\tilde{d}\right) \frac{\partial}{\partial x}\left[w^{\prime}(x) \psi\right]-\left(\tilde{d}_{n p}+\tilde{d}\right) \frac{\partial}{\partial x}\left[w(x) \frac{\partial \psi}{\partial x}\right], \\
& L_{22} \varphi=\left(\bar{g}_{n p}+\bar{g}\right) \frac{\partial^{2}[w(x) \varphi]}{\partial x^{2}} .
\end{aligned}
$$

When $\bar{g}>\bar{g}_{n p}$, the asymptotic stability of system (2.43) (i.e. the equations (2.40) and $(2.41)$ ) is proved in Corollary 4.2 (see Section 4). Thus the equations (2.38) and (2.39) have asymptotic stability if the condition $\bar{g}>\bar{g}_{n p}$ holds. However, if $g_{n n}=$ $g_{p p}=g_{n p}=0$, then system (2.43) becomes $\psi_{t}=f_{1}$ and $\varphi_{t}=f_{2}$, which imply $(\psi, \varphi)=$ $\left(\psi_{0}+t f_{1}, \varphi_{0}+t f_{2}\right)$ for $t>0$, where $\psi_{0}=\left.\psi\right|_{t=0}$ and $\varphi_{0}=\left.\varphi\right|_{t=0}$ are initial conditions of $\psi$ and $\varphi$, respectively. Hence the asymptotic stability of system (2.43) is gone. This shows that the effect of coupling constants $g_{i j}$ 's changes the asymptotic stability of system (2.43).

Note that the operator $\left(\begin{array}{ll}L_{11} & L_{12} \\ L_{21} & L_{22}\end{array}\right)=\left(\begin{array}{cc}L_{11} & 0 \\ 0 & L_{22}\end{array}\right)$ becomes diagonal if $\tilde{d}_{n p}-\tilde{d}=$ $\tilde{d}_{n p}+\tilde{d}=0$, i.e. $\tilde{d}_{n p}=\tilde{d}=0$. By $(2.42)$, the condition $\tilde{d}_{n p}=\tilde{d}=0$ is equivalent to $D_{p} z_{p}+$ $D_{n} z_{n}=0$ and $D_{n} z_{p} g_{n n}+D_{p} z_{n} g_{p p}=0$, being fulfilled if $D_{p}=D_{n}, z_{p}=-z_{n}$, and $g_{n n}=$ $g_{p p}$. In Section 5, the case of $D_{p}=D_{n}=D>0, z_{p}=-z_{n}=z>0$, and $g_{n n}=g_{p p}=g>0$ (i.e. symmetry electrolytes) is considered in order to get the instability condition $g<g_{n p}$.

\section{The approximate LJ potential}

In this section, we study the approximation of the functional $E_{L J}$ defined in (1.2) and written as follows:

$$
E_{L J}\left[c_{i}, c_{j}\right]=\lim _{\sigma \rightarrow 0} \iint_{\mathbb{R}^{d} \times \mathbb{R}^{d}}\left[\left(\Psi_{12}-\Psi_{6}\right) \chi_{\sigma}\right](x-y) c_{i}(x) c_{j}(y) d x d y,
$$

for $c_{i}, c_{j} \in L_{+}^{2}\left(\mathbb{R}^{d}\right)=\left\{f \in L^{2}\left(\mathbb{R}^{d}\right): f \geq 0\right\}$. Here $\Psi_{12}(z)=C_{1}|z|^{-12}$ is the repulsion term and $\Psi_{6}(z)=C_{2}|z|^{-6}$ is the attraction term of the LJ potential, and $\chi_{\sigma}=\chi_{\sigma}(z)$ is the characteristic function of the exterior ball $\left\{z \in \mathbb{R}^{d}:|z|>\sigma\right\}$ satisfying

$$
\chi_{\sigma}(z)=\left\{\begin{array}{ccc}
1, & \text { if } & |z|>\sigma \\
0, & \text { if } & |z| \leq \sigma
\end{array}\right.
$$

The main idea here is to approximate $\widehat{\Psi_{m} \chi_{\sigma}}(\xi)(m=6,12)$, the Fourier transform of the kernel $\Psi_{m} \chi_{\sigma}$, by $\widehat{\Psi_{m} \chi_{\sigma}} \hat{\varphi}_{\sigma}(\xi)$ in the frequency space, where the hat denotes Fourier transform and $\varphi_{\sigma}$ is a band-limited function satisfying

$$
\hat{\varphi}_{\sigma}(\xi)=1-\chi_{\sigma^{-\gamma}}(\xi) \text { for } \xi \in \mathbb{R}^{d} .
$$


Here $1-\chi_{\sigma^{-\gamma}}$ is defined by

$$
1-\chi_{\sigma^{-\gamma}}(\xi)=\left\{\begin{array}{rrr}
1, & \text { if } & |\xi| \leq \sigma^{-\gamma} \\
0, & \text { if } & |\xi|>\sigma^{-\gamma}
\end{array}\right.
$$

where $\sigma>0$ is a small parameter tending to zero, and $0<\gamma<1$ is a constant independent of $\xi$ and $\sigma$. Note that $1-\chi_{\sigma^{-\gamma}}$ denotes the characteristic function of the ball $\left\{\xi \in \mathbb{R}^{d}:|\xi| \leq \sigma^{-\gamma}\right\}$, which expands to the entire space $\mathbb{R}^{d}$ as $\sigma$ goes to zero.

By the standard formulas of Fourier analysis, it is obvious that

$$
\widehat{\Psi_{m} \chi_{\sigma}}(\xi) \hat{\varphi}_{\sigma}(\xi)=\left[\left(\widetilde{\left.\Psi_{m} \chi_{\sigma}\right) \star \varphi_{\sigma}}\right](\xi),\right.
$$

for $\xi \in \mathbb{R}^{d}$, where the asterisk denotes convolution. The approximate kernel of $\Psi_{m}$ is given as follows:

$$
K_{m, \sigma}(z)=\left(\Psi_{m} \chi_{\sigma}\right) \star \varphi_{\sigma}(z) \quad \text { for } \quad z \in \mathbb{R}^{d}, \quad m=6,12,
$$

and the approximate energy functional $E_{L J, \sigma}$ is given by

$$
E_{L J, \sigma}\left[c_{i}, c_{j}\right]:=\iint_{\mathbb{R}^{d} \times \mathbb{R}^{d}}\left(K_{12, \sigma}-K_{6, \sigma}\right)(x-y) c_{i}(x) c_{j}(y) d x d y,
$$

for $c_{i}, c_{j} \in L_{+}^{2}\left(\mathbb{R}^{d}\right)$. By standard theorems of Fourier analysis (cf. [54]), it is easy to check that the functions $\Psi_{m} \chi_{\sigma}$ and $\varphi_{\sigma}$ are of $L^{2}\left(\mathbb{R}^{d}\right) \cap L^{\infty}\left(\mathbb{R}^{d}\right)$, which implies $K_{m, \sigma} \in L^{2}\left(\mathbb{R}^{d}\right) \cap L^{\infty}\left(\mathbb{R}^{d}\right)$. The following proposition is for the approximation of $E_{L J, \sigma}$ to $E_{L J}$.

Proposition 3.1. Assume $c_{i}, c_{j} \in L_{+}^{2}\left(\mathbb{R}^{d}\right)$ satisfy the following hypothesis:

(H) $\lim _{\sigma \rightarrow 0+}\left[\int_{\mathbb{R}^{d}} \widehat{\Psi \chi_{\sigma}}(\xi)\left[1-\chi_{\sigma^{-\gamma}}(\xi)\right] \widehat{c_{i}}(\xi) \widehat{c_{j}}(\xi) d \xi-\int_{\mathbb{R}^{d}} \widehat{\Psi \chi_{\sigma}}(\xi) \widehat{c_{i}}(\xi) \widehat{c_{j}}(\xi) d \xi\right]=0$, where $0<\gamma<1$ is a constant independent of $\sigma$ and $\xi$. Then

$$
\lim _{\sigma \rightarrow 0+}\left\{E_{L J, \sigma}\left[c_{i}, c_{j}\right]-E_{L J}\left[c_{i}, c_{j}\right]\right\}=0 .
$$

Note that the hypothesis $(\mathrm{H})$ is achievable at least for functions $c_{i}$ satisfying $\widehat{c_{i}}(\xi)=0$ for $|\xi|>\sigma^{-\gamma}$ and $i=1, \cdots, N$, which means all the high frequencies of $c_{i}$ have been cut off. Due to the strong singularity of $\Psi, \widehat{\Psi \chi_{\sigma}}(\xi)$ has no asymptotic behavior like the right side of (3.12) as $\sigma$ goes to zero, especially for $|\xi| \sim \sigma^{-1}$ (see Remark 3.3). This motivates us to replace $\widehat{\Psi \chi_{\sigma}}(\xi)$ by $\widehat{\Psi \chi_{\sigma}}(\xi)\left[1-\chi_{\sigma^{-\gamma}}(\xi)\right]$, which is a kind of truncation on the $\xi$ variable and has a simple asymptotic formula (3.12). The truncation may lose the effect of high frequencies $|\xi|>\sigma^{-\gamma}(0<\gamma<1)$, but still involve a large part of the steric effects because it keeps the effect of frequencies like $|\xi| \sim \sigma^{-\alpha}$ for $0<\alpha<\gamma$ tending to infinity as $\sigma$ goes to zero. Please note that the main goal of our work is to simplify the model of Liu and Eisenberg [16, 28]. Here we present a simplified model which is easy to study and useful to understand the selectivity of ion channels [25].

For the proof of Proposition 3.1, we use the Fourier transform to calculate the integral

$$
\iint_{\mathbb{R}^{d} \times \mathbb{R}^{d}} \Psi_{m} \chi_{\sigma}(|x-y|) c_{i}(x) c_{j}(y) d x d y
$$


and get

$$
\begin{aligned}
\iint_{\mathbb{R}^{d} \times \mathbb{R}^{d}} \Psi_{m} \chi_{\sigma}(|x-y|) c_{i}(x) c_{j}(y) d x d y & =\int_{\mathbb{R}^{d}}\left[\left(\Psi_{m} \chi_{\sigma}\right) \star c_{j}\right](x) c_{i}(x) d x \\
& =\int_{\mathbb{R}^{d}}\left[\left(\Psi_{m} \widehat{\chi_{\sigma}}\right) \star c_{j}\right](\xi) \widehat{c_{i}}(\xi) d \xi \\
& =\int_{\mathbb{R}^{d}} \widehat{\Psi_{m} \chi_{\sigma}}(\xi) \widehat{c_{i}}(\xi) \widehat{c_{j}}(\xi) d \xi,
\end{aligned}
$$

i.e.

$$
\iint_{\mathbb{R}^{d} \times \mathbb{R}^{d}} \Psi_{m} \chi_{\sigma}(|x-y|) c_{i}(x) c_{j}(y) d x d y=\int_{\mathbb{R}^{d}} \widehat{\Psi_{m} \chi_{\sigma}}(\xi) \widehat{c_{i}}(\xi) \widehat{c_{j}}(\xi) d \xi
$$

On the other hand, (3.2)-(3.4) give

$$
\begin{aligned}
\iint_{\mathbb{R}^{d} \times \mathbb{R}^{d}} K_{m, \sigma}(x-y) c_{i}(x) c_{j}(y) d x d y & =\int_{\mathbb{R}^{d}}\left(K_{m, \sigma} \star c_{j}\right)(x) c_{i}(x) d x \\
& =\int_{\mathbb{R}^{d}}\left(\widehat{K_{m, \sigma} \star c_{j}}\right)(\xi) \widehat{c_{i}}(\xi) d \xi \\
& =\int_{\mathbb{R}^{d}} \widehat{K_{m, \sigma}}(\xi) \widehat{c_{i}}(\xi) \widehat{c_{j}}(\xi) d \xi \\
& =\int_{\mathbb{R}^{d}}\left[\left(\widehat{\Psi_{m} \chi_{\sigma}}\right) \star \varphi_{\sigma}\right](\xi) \widehat{c_{i}}(\xi) \widehat{c_{j}}(\xi) d \xi \\
& =\int_{\mathbb{R}^{d}} \widehat{\Psi_{m} \chi_{\sigma}}(\xi) \hat{\varphi}_{\sigma}(\xi) \widehat{c_{i}}(\xi) \widehat{c_{j}}(\xi) d \xi \\
& =\int_{\mathbb{R}^{d}} \widehat{\Psi_{m} \chi_{\sigma}}(\xi)\left[1-\chi_{\sigma}-\gamma(\xi)\right] \widehat{c_{i}}(\xi) \widehat{c_{j}}(\xi) d \xi
\end{aligned}
$$

i.e.

$$
\iint_{\mathbb{R}^{d} \times \mathbb{R}^{d}} K_{m, \sigma}(x-y) c_{i}(x) c_{j}(y) d x d y=\int_{\mathbb{R}^{d}} \widehat{\Psi_{m} \chi_{\sigma}}(\xi)\left[1-\chi_{\sigma^{-\gamma}}(\xi)\right] \widehat{c_{i}}(\xi) \widehat{c_{j}}(\xi) d \xi .
$$

Here Parseval's formula and the convolution theorem for the Fourier transform (cf. [39]) have been used because $c_{i}, c_{j} \in L_{+}^{2}\left(\mathbb{R}^{d}\right)$. Thus the hypothesis (H), (3.7), and (3.8) imply that

$\lim _{\sigma \rightarrow 0+}\left[\iint_{\mathbb{R}^{d} \times \mathbb{R}^{d}} K_{m, \sigma}(x-y) c_{i}(x) c_{j}(y) d x d y-\iint_{\mathbb{R}^{d} \times \mathbb{R}^{d}} \Psi_{m}(|x-y|) c_{i}(x) c_{j}(y) d x d y\right]=0$,

and $K_{m, \sigma}$ can be regarded as an approximation of the kernel $\Psi_{m}$ for $m=6,12$. Combining (3.1), (3.5), and (3.9), it is obvious that

$$
\begin{aligned}
& \lim _{\sigma \rightarrow 0+}\left(E_{L J, \sigma}-E_{L J}\right) \\
= & \sum_{m=6,12} \lim _{\sigma \rightarrow 0+}\left[\iint_{\mathbb{R}^{d} \times \mathbb{R}^{d}} K_{m, \sigma}(x-y) c_{i}(x) c_{j}(y) d x d y-\iint_{\mathbb{R}^{d} \times \mathbb{R}^{d}} \Psi_{m}(|x-y|) c_{i}(x) c_{j}(y) d x d y\right] \\
= & 0,
\end{aligned}
$$

so (3.6) holds and the proof of Proposition 3.1 is complete. 
As $\sigma$ goes to zero, the asymptotic behavior of the functional $E_{L J, \sigma}$ is stated as follows.

Proposition 3.2. Under the same hypotheses of Proposition 3.1,

$$
E_{L J, \sigma}\left[c_{i}, c_{j}\right]=C_{1} \frac{\omega_{d}}{12-d} \sigma^{d-12}\left(1+o_{\sigma}(1)\right) \int_{\mathbb{R}^{d}} c_{i}(x) c_{j}(x) d x,
$$

where $\omega_{d}$ is the surface area of the d-dimensional unit ball and $o_{\sigma}(1)$ is a small quantity tending to zero as $\sigma$ goes to zero.

Proof. Using the definition of the Fourier transform, the function $\widehat{\Psi_{m} \chi_{\sigma}}(\xi)\left[1-\chi_{\sigma^{-\gamma}}(\xi)\right]$ can be expressed by

$$
\widehat{\Psi_{m} \chi_{\sigma}}(\xi)\left[1-\chi_{\sigma^{-\gamma}}(\xi)\right]=\alpha_{m} \int_{|x|>\sigma} \exp \{\sqrt{-1} x \cdot \xi\}|x|^{-m} d x\left[1-\chi_{\sigma^{-\gamma}}(\xi)\right]
$$

for $m=6,12$, where $\alpha_{m}=C_{1}$ if $m=12, \alpha_{m}=C_{2}$ if $m=6$. Let $\tilde{\xi}=\sigma^{\gamma} \xi$ and $\tilde{x}=\sigma^{-\gamma} x$. Then $|\xi| \leq \sigma^{-\gamma}$ is equivalent to $|\tilde{\xi}| \leq 1,|x|>\sigma$ is equivalent to $|\tilde{x}|>\sigma^{1-\gamma}$. Besides, $|x|^{-m}=\sigma^{-m \gamma}|\tilde{x}|^{-m}, d x=\sigma^{d \gamma} d \tilde{x}$, and hence

$$
\begin{aligned}
\widehat{\Psi_{m} \chi_{\sigma}}(\xi)\left[1-\chi_{\sigma^{-\gamma}}(\xi)\right] & =\alpha_{m}\left[1-\chi_{\sigma^{-\gamma}}(\xi)\right] \int_{|x|>\sigma} \exp \{\sqrt{-1} x \cdot \xi\}|x|^{-m} d x \\
& =\alpha_{m} \sigma^{(d-m) \gamma}\left[1-\chi_{1}(\tilde{\xi})\right] \int_{|\tilde{x}|>\sigma^{1-\gamma}} \exp \{\sqrt{-1} \tilde{x} \cdot \tilde{\xi}\}|\tilde{x}|^{-m} d \tilde{x} .
\end{aligned}
$$

Note that $0<\gamma<1,1-\chi_{1}(\tilde{\xi})=0$ if $|\tilde{\xi}|>1$, and $1-\chi_{1}(\tilde{\xi})=1$ if $|\tilde{\xi}| \leq 1$. Then

$$
\left[1-\chi_{1}(\tilde{\xi})\right] \int_{|\tilde{x}|>\sigma^{1-\gamma}} \exp \{\sqrt{-1} \tilde{x} \cdot \tilde{\xi}\}|\tilde{x}|^{-m} d \tilde{x}=\frac{\omega_{d}}{m-d} \sigma^{(d-m)(1-\gamma)}\left(1+o_{\sigma}(1)\right),
$$

i.e.

$$
\widehat{\Psi_{m} \chi} \sigma(\xi)\left[1-\chi_{\sigma^{-\gamma}}(\xi)\right]=\frac{\omega_{d}}{m-d} \sigma^{d-m}\left(1+o_{\sigma}(1)\right),
$$

where $\omega_{d}$ is the surface area of the $d$-dimensional unit ball and $o_{\sigma}(1)$ is a small quantity tending to zero as $\sigma$ goes to zero. Thus (3.8), (3.12), and Parseval's formula for the Fourier transform (cf. [39]) imply

$$
\begin{aligned}
\iint_{\mathbb{R}^{d} \times \mathbb{R}^{d}} K_{m, \sigma}(x-y) c_{i}(x) c_{j}(y) d x d y & =\alpha_{m} \frac{\omega_{d}}{m-d} \sigma^{d-m}\left(1+o_{\sigma}(1)\right) \int_{\mathbb{R}^{d}} \widehat{c_{i}}(\xi) \widehat{c_{j}}(\xi) d \xi \\
& =\alpha_{m} \frac{\omega_{d}}{m-d} \sigma^{d-m}\left(1+o_{\sigma}(1)\right) \int_{\mathbb{R}^{d}} c_{i}(x) c_{j}(x) d x,
\end{aligned}
$$

for $m=6,12$. Therefore, the proof of (3.10) is complete.

Remark 3.3. Suppose $\gamma=1$. Then the asymptotic behaviors (3.11) may fail. Hence the condition $0<\gamma<1$ can not be generalized to $\gamma=1$.

Combining Proposition 3.1, (3.6), and (3.10), we have the following obvious theorem.

TheOREM 3.4. Under the same hypotheses of Proposition 3.1, the energy functional $E_{L J}$ satisfies

$$
E_{L J}\left[c_{i}, c_{j}\right]=C_{1} \frac{\omega_{d}}{12-d} \sigma^{d-12}\left(1+o_{\sigma}(1)\right) \int_{\mathbb{R}^{d}} c_{i}(x) c_{j}(x) d x,
$$


as $\sigma$ goes to zero, where $\omega_{d}$ is the surface area of the $d$-dimensional unit ball and $o_{\sigma}(1)$ is a small quantity tending to zero as $\sigma$ goes to zero.

\section{Stability conditions}

Let $(\psi, \varphi)$ be a smooth solution of the system $(2.43)$ with the zero Dirichlet boundary condition $\psi=\varphi=0$ at $x= \pm 1$. Note that the function $\phi_{0}$ is a smooth function. We take the $L_{x}^{2}$-inner product of $(2.43)$ and $\left(\begin{array}{l}\psi \\ \varphi\end{array}\right)$, and then we may use (2.46)-(2.49) and integration by parts to get

$$
\frac{d}{d t} \int_{-1}^{1}\left(\psi^{2}+\varphi^{2}\right) d x=I+I I+I I I,
$$

where $\psi_{x}=\frac{\partial \psi}{\partial x}, \varphi_{x}=\frac{\partial \varphi}{\partial x},(\cdot)_{x}=\frac{\partial}{\partial x}$, and

$$
\begin{gathered}
I=\int_{-1}^{1}\left(f_{1} \psi+f_{2} \varphi\right) d x \\
I I=-\left(\bar{g}+\bar{g}_{n p}\right) \int_{-1}^{1} w^{\prime}(x) \psi \psi_{x} d x-\left(\bar{g}-\bar{g}_{n p}\right) \int_{-1}^{1} w \psi_{x}^{2} d x-\left(\bar{g}_{n p}+\bar{g}\right) \int_{-1}^{1}(w \varphi)_{x} \varphi_{x} d x \\
I I I=-\left(\tilde{d}_{n p}-\tilde{d}\right) \int_{-1}^{1}(w \varphi)_{x} \psi_{x} d x \\
\quad-\left(\tilde{d}_{n p}-\tilde{d}\right) \int_{-1}^{1} w^{\prime}(x) \psi \varphi_{x} d x+\left(\tilde{d}_{n p}+\tilde{d}\right) \int_{-1}^{1} w \psi_{x} \varphi_{x} d x .
\end{gathered}
$$

Using integration by parts and the zero Dirichlet boundary conditions $\psi=0$ at $x= \pm 1$, it is obvious that

$$
\int_{-1}^{1} w^{\prime}(x) \psi \psi_{x} d x=\int_{-1}^{1} \frac{1}{2} w^{\prime}(x)\left(\psi^{2}\right)_{x} d x=-\frac{1}{2} \int_{-1}^{1} w^{\prime \prime}(x) \psi^{2} d x
$$

Similarly, due to $\varphi=0$ at $x= \pm 1$,

$$
\int_{-1}^{1} w^{\prime}(x) \varphi \varphi_{x} d x=-\frac{1}{2} \int_{-1}^{1} w^{\prime \prime}(x) \varphi^{2} d x
$$

and then

$$
\begin{aligned}
\int_{-1}^{1}(w \varphi)_{x} \varphi_{x} d x & =\int_{-1}^{1} w\left|\varphi_{x}\right|^{2} d x+\int_{-1}^{1} w^{\prime}(x) \varphi \varphi_{x} d x \\
& =\int_{-1}^{1} w\left|\varphi_{x}\right|^{2} d x-\frac{1}{2} \int_{-1}^{1} w^{\prime \prime}(x) \varphi^{2} d x
\end{aligned}
$$

Hence II becomes

$$
\begin{array}{r}
I I=-\left(\bar{g}-\bar{g}_{n p}\right) \int_{-1}^{1} w \psi_{x}^{2} d x+\frac{1}{2}\left(\bar{g}+\bar{g}_{n p}\right) \int_{-1}^{1} w^{\prime \prime}(x) \psi^{2} d x \\
-\left(\bar{g}_{n p}+\bar{g}\right)\left[\int_{-1}^{1} w\left|\varphi_{x}\right|^{2} d x-\frac{1}{2} \int_{-1}^{1} w^{\prime \prime}(x) \varphi^{2} d x\right] .
\end{array}
$$

By (2.32), the solution $w$ satisfies

$$
B \geq w(x)=\frac{\sqrt{\left(B_{1}^{2}-B_{2}^{2}\right) x+B_{1}^{2}+B_{2}^{2}}}{\sqrt{2}\left|z_{n} z_{p}\right|} \geq b>0 \quad \text { for } \quad x \in[-1,1],
$$


and $w^{\prime \prime}(x) \leq 0$ for $x \in(-1,1)$, where $b=\frac{\min \left\{B_{1}, B_{2}\right\}}{\left|z_{n} z_{p}\right|}$ and $B=\frac{\max \left\{B_{1}, B_{2}\right\}}{\left|z_{n} z_{p}\right|}$ are positive constants. Suppose $\bar{g}>\bar{g}_{n p}$. Then (4.3) and (4.4) imply

$$
I I \leq-b\left(\bar{g}-\bar{g}_{n p}\right) \int_{-1}^{1}\left|\psi_{x}\right|^{2} d x-b\left(\bar{g}_{n p}+\bar{g}\right) \int_{-1}^{1}\left|\varphi_{x}\right|^{2} d x,
$$

Here we have used the fact that $\bar{g}$ and $\bar{g}_{n p}$ are positive constants. By the H"older inequality, it is obvious that

$$
I \leq C_{1}\left[\int_{-1}^{1}\left(\psi^{2}+\varphi^{2}\right) d x\right]^{1 / 2}
$$

where $C_{1}$ is a positive constant depending on $f_{1}$ and $f_{2}$. On the other hand, both $\psi$ and $\varphi$ satisfy the Sobolev inequalities

$$
\int_{-1}^{1} \psi^{2} d x \leq C_{2} \int_{-1}^{1}\left|\psi_{x}\right|^{2} d x
$$

and

$$
\int_{-1}^{1} \varphi^{2} d x \leq C_{2} \int_{-1}^{1}\left|\varphi_{x}\right|^{2} d x
$$

where $C_{2}$ is a positive constant independent of $\psi$ and $\varphi$. Hence by (4.4), (4.7), (4.8), and the Hölder inequality, we get

$$
I I I \leq\left(\left|\tilde{d}_{n p}\right|+|\tilde{d}|\right) C_{3} \int_{-1}^{1}\left(\left|\psi_{x}\right|^{2}+\left|\varphi_{x}\right|^{2}\right) d x
$$

where $C_{3}$ is a positive constant independent of $\tilde{d}, \tilde{d}_{n p}, \psi$, and $\varphi$. Suppose both $|\tilde{d}|$ and $\left|\tilde{d}_{n p}\right|$ are sufficiently small such that $\left(\left|\tilde{d}_{n p}\right|+|\tilde{d}|\right) C_{3}<b\left(\bar{g}-\bar{g}_{n p}\right)$. Then (4.5) and (4.9) give

$$
I I+I I I \leq-\theta \int_{-1}^{1}\left(\left|\psi_{x}\right|^{2}+\left|\varphi_{x}\right|^{2}\right) d x
$$

where $\theta=b\left(\bar{g}-\bar{g}_{n p}\right)-\left(\left|\tilde{d}_{n p}\right|+|\tilde{d}|\right) C_{3}$ is a positive constant. Consequently, (4.7), (4.8), and (4.10) imply

$$
I I+I I I \leq-\theta_{0} \int_{-1}^{1}\left(\psi^{2}+\varphi^{2}\right) d x
$$

where $\theta_{0}=\frac{\theta}{C_{2}}$ is a positive constant. Let $M(t)=\int_{-1}^{1}\left(\psi^{2}+\varphi^{2}\right) d x$ for $t>0$. Then combining (4.1), (4.6), and (4.11), we have

$$
\frac{d M}{d t} \leq C_{1} \sqrt{M}-\theta_{0} M \quad \text { for } \quad t>0 .
$$

Notice that as $M>\left(C_{1} / \theta_{0}\right)^{2}$, the right side of (4.12) becomes negative and $\frac{d M}{d t}<0$. This implies that $\sup _{t>0} M(t)<\infty$, and the result can be summarized as follows. 
THEOREM 4.1. Let $(\psi, \varphi)$ be a smooth solution of the system (2.43) with the zero Dirichlet boundary condition $\psi=\varphi=0$ at $x= \pm 1$. Suppose $\bar{g}>\bar{g}_{n p}$, where both $\bar{g}$ and $\bar{g}_{n p}$ are defined in (2.42). Assume both $|\tilde{d}|$ and $\left|\tilde{d}_{n p}\right|$ are sufficiently small such that $\left(\left|\tilde{d}_{n p}\right|+|\tilde{d}|\right) C_{3}<b\left(\bar{g}-\bar{g}_{n p}\right)$, where $b$ and $C_{3}$ are given in (4.4) and (4.9), respectively. Then the function $M(t)=\int_{-1}^{1}\left(\psi^{2}+\varphi^{2}\right) d x$ is uniformly bounded in $t>0$, i.e. $\sup _{t>0} M(t)<\infty$.

Now we want to show that the condition $\bar{g}>\bar{g}_{n p}$ gives the asymptotic stability of the solution of the system (2.43) with the zero Dirichlet boundary condition $\psi=\varphi=0$ at $x= \pm 1$. Here the asymptotic stability means that $\sup _{t>0}\|\psi\|_{L_{x}^{2}}+\|\varphi\|_{L_{x}^{2}}<\infty$ and $\left\|\psi-\psi_{\infty}\right\|_{L_{x}^{2}}+\left\|\varphi-\varphi_{\infty}\right\|_{L_{x}^{2}} \rightarrow 0$ as $t \rightarrow \infty$, whenever $(\psi, \varphi)$ is a (weak) solution of the system (2.43) with the zero Dirichlet boundary condition $\psi=\varphi=0$ at $x= \pm 1$ and various initial conditions $\left.(\psi, \varphi)\right|_{t=0}=\left(\psi_{0}, \varphi_{0}\right)$, where $\left(\psi_{\infty}, \varphi_{\infty}\right)$ is the steady state of the system (2.43) satisfying

$$
\left(\begin{array}{ll}
L_{11} & L_{12} \\
L_{21} & L_{22}
\end{array}\right)\left(\begin{array}{l}
\psi_{\infty} \\
\varphi_{\infty}
\end{array}\right)=\left(\begin{array}{l}
-f_{1} \\
-f_{2}
\end{array}\right)
$$

Note that the uniform boundedness of $\|\psi\|_{L_{x}^{2}}+\|\varphi\|_{L_{x}^{2}}$ has been obtained by Theorem 4.1. To get the asymptotic stability, it is sufficient to show that $\left\|\psi-\psi_{\infty}\right\|_{L_{x}^{2}}+$ $\left\|\varphi-\varphi_{\infty}\right\|_{L_{x}^{2}} \rightarrow 0$ as $t \rightarrow \infty$. Let $\Psi=\psi-\psi_{\infty}$ and $\Phi=\varphi-\varphi_{\infty}$, where $(\psi, \varphi)$ is the solution of the system (2.43) with the zero Dirichlet boundary condition $\psi=\varphi=0$ at $x= \pm 1$ and the initial condition $\left.(\psi, \varphi)\right|_{t=0}=\left(\psi_{0}, \varphi_{0}\right)$. Then (2.43) implies that $(\Psi, \Phi)$ satisfies

$$
\frac{\partial}{\partial t}\left(\begin{array}{c}
\Psi \\
\Phi
\end{array}\right)=\left(\begin{array}{ll}
L_{11} & L_{12} \\
L_{21} & L_{22}
\end{array}\right)\left(\begin{array}{c}
\Psi \\
\Phi
\end{array}\right)
$$

with the zero Dirichlet boundary condition $\Psi=\Phi=0$ at $x= \pm 1$ and the initial condition

$$
\left.(\Psi, \Phi)\right|_{t=0}=\left(\Psi_{0}, \Phi_{0}\right), \quad \Psi_{0}=\psi_{0}-\psi_{\infty}, \quad \Phi_{0}=\varphi_{0}-\varphi_{\infty} .
$$

Then as for (4.12), we may use (4.13), i.e. (2.43) with $f_{1} \equiv f_{2} \equiv 0$, to derive

$$
\frac{d \bar{M}}{d t} \leq-\theta_{0} \bar{M} \quad \text { for } \quad t>0
$$

where $\bar{M}=\int_{-1}^{1} \Psi^{2}+\Phi^{2} d x$. Consequently, $\bar{M}(t) \leq \bar{M}(0) e^{-\theta_{0} t}$ for $t>0$, and then $\bar{M}(t) \rightarrow 0$ as $t \rightarrow \infty$. This shows the asymptotic stability of the solution of the system (2.43) with the zero Dirichlet boundary condition $\psi=\varphi=0$ at $x= \pm 1$. Therefore, we have proved the following.

Corollary 4.2. Suppose $\bar{g}>\bar{g}_{n p}$, where both $\bar{g}$ and $\bar{g}_{n p}$ are defined in (2.42). Assume both $|\tilde{d}|$ and $\left|\tilde{d}_{n p}\right|$ are sufficiently small such that $\left(\left|\tilde{d}_{n p}\right|+|\tilde{d}|\right) C_{3}<b\left(\bar{g}-\bar{g}_{n p}\right)$, where $b$ and $C_{3}$ are given in (4.4) and (4.9), respectively. Then the system (2.43) with the zero Dirichlet boundary condition becomes asymptotically stable. 


\section{Instability conditions}

Assume $z_{p}=-z_{n}=z>0$ (i.e. symmetry electrolytes), $D_{n}=D_{p}=D>0$, and $g_{n n}=$ $g_{p p}=g>0$. Then the coefficients $\bar{g}_{n p}, \tilde{d}_{n p}, \bar{g}$, and $\tilde{d}$ (defined in (2.42)) become

$$
\left\{\begin{array}{l}
\bar{g}_{n p}=D z g_{n p}, \\
\bar{g}=D z g \\
\tilde{d}_{n p}=\tilde{d}=0 .
\end{array}\right.
$$

Hence the conditions of Corollary 4.2 can be fulfilled and the system (2.43) with the zero Dirichlet boundary condition becomes asymptotically stable if $g>g_{n p}$ holds. Nevertheless, one may not know whether the opposite condition $g<g_{n p}$ changes the stability or not. In the rest of the section, it is proved that under the condition $g<g_{n p}$, the system (2.43) with the zero Dirichlet boundary condition becomes unstable for some specific initial data. Instability is of great interest because it may be related to the spontaneous gating phenomena seen whenever currents through single ion channels are measured [13, 43, 44, 49].

By (2.34), the electric potential $\phi_{0}$ has the following simple form:

$$
\phi_{0}(x)=a_{0} x+b_{0} \quad \text { for } \quad x \in(-1,1),
$$

where $a_{0}$ and $b_{0}$ are constants. By (5.1), the system (2.43) can be transformed into

$$
\frac{\partial}{\partial t}\left(\begin{array}{c}
\psi \\
\varphi
\end{array}\right)=\left(\begin{array}{c}
2 z^{3} D\left(w \phi_{0}{ }^{\prime}\right)^{\prime} \\
2 z^{2} D w^{\prime \prime}
\end{array}\right)+\left(\begin{array}{c}
z D\left(g+g_{n p}\right) \frac{\partial}{\partial x}\left(w^{\prime} \psi\right)+z D\left(g-g_{n p}\right) \frac{\partial}{\partial x}\left(w \frac{\partial \psi}{\partial x}\right) \\
z D\left(g+g_{n p}\right) \frac{\partial^{2}}{\partial x^{2}}(w \varphi)
\end{array}\right),
$$

and also may be decomposed into two independent equations as follows:

$$
\frac{1}{z D} \frac{\partial \psi}{\partial t}=2 z^{2}\left(w \phi_{0}^{\prime}\right)^{\prime}+\left(g+g_{n p}\right) \frac{\partial}{\partial x}\left(w^{\prime} \psi\right)+\left(g-g_{n p}\right) \frac{\partial}{\partial x}\left(w \frac{\partial \psi}{\partial x}\right)
$$

and

$$
\frac{1}{z D} \frac{\partial \varphi}{\partial t}=2 z w^{\prime \prime}+\left(g+g_{n p}\right) \frac{\partial^{2}}{\partial x^{2}}(w \varphi)
$$

for $x \in(-1,1)$ and $t>0$. Note that both (5.3) and (5.4) are non-homogeneous parabolic partial differential equations. To simplify the form of (5.3), let

$$
\tilde{\psi}(x, t)=e^{-h(x)} \psi(x, t), \quad \text { i.e. } \quad \psi(x, t)=e^{h(x)} \tilde{\psi}(x, t),
$$

for $x \in(-1,1)$, and $t>0$, where $h(x)=\alpha_{0} \ln w(x)$ and $\alpha_{0}=\frac{g_{n p}+g}{g_{n p}-g}>0$ because $g_{n p}>$ $g>0$. Note that $w(x)>0$ for $x \in(-1,1)$ so the function $h$ is well-defined. Moreover, because the function $w$ has positive upper and lower bounds defined in (4.4), the function $e^{h(x)}=w(x)^{\alpha_{0}}$ also has positive upper and lower bounds as follows:

$$
0<B_{1} \leq e^{h(x)} \leq B_{2} \quad \text { for } \quad x \in(-1,1)
$$

where $B_{1}$ and $B_{2}$ are positive constants. Thus by (5.5) and $\alpha_{0}=\frac{g_{n p}+g}{g_{n p}-g}>0$, the equation (5.3) can be transformed into

$$
\frac{1}{z D} e^{h} \frac{\partial \tilde{\psi}}{\partial t}=2 z^{2}\left(w \phi_{0}^{\prime}\right)^{\prime}+\left(g-g_{n p}\right) \frac{\partial}{\partial x}\left(w e^{h} \frac{\partial \tilde{\psi}}{\partial x}\right) \quad \text { for } \quad x \in(-1,1), t>0 .
$$


Note that $h=\alpha_{0} \ln w$ implies $w h^{\prime}=\alpha_{0} w^{\prime}$.

The equation (5.7) also can be written as

$$
\frac{1}{z D} e^{h} \frac{\partial \tilde{\psi}}{\partial t}=2 z^{2}\left(w \phi_{0}^{\prime}\right)^{\prime}+\left(g_{n p}-g\right) L \tilde{\psi}
$$

where $L$ is a linear differential operator defined by

$$
L u=-\frac{d}{d x}\left(w e^{h} \frac{d u}{d x}\right) \quad \text { for } \quad u=u(x) \in H^{2}((-1,1)) \cap H_{0}^{1}((-1,1)) .
$$

It is obvious that the operator $L$ is self-adjoint. Because $w$ is a positive function, integration by parts gives

$$
\begin{aligned}
\int_{-1}^{1} u L u d x & =-\int_{-1}^{1} u \frac{d}{d x}\left(w e^{h} \frac{d u}{d x}\right) d x \\
& =\int_{-1}^{1} w e^{h}\left(\frac{d u}{d x}\right)^{2} d x>0
\end{aligned}
$$

for $u \neq \equiv 0$ and $u \in H^{2}((-1,1)) \cap H_{0}^{1}((-1,1))$, and hence the operator $L$ is positive. To solve the equation (5.8), the following weighted eigenvalue problem is considered:

$$
\left\{\begin{array}{l}
L \zeta=\lambda e^{h} \zeta, \quad \text { in }(-1,1), \\
\zeta( \pm 1)=0,
\end{array}\right.
$$

where $\lambda$ is the eigenvalue and $\zeta$ is the corresponding eigenfunction. Because the operator $L$ is self-adjoint and positive, it is well-known that the eigenvalue problem (5.10) has eigenvalues $\left\{\lambda_{j}\right\}_{j=1}^{\infty}$ and the associated eigenfunctions $\left\{\zeta_{j}\right\}_{j=1}^{\infty}$ such that

$$
0<\lambda_{1} \leq \lambda_{2} \leq \lambda_{3} \leq \cdots
$$

and $\left\{\zeta_{j}\right\}_{j=1}^{\infty}$ forms an orthonormal basis of the following weighted $L^{2}$ function space defined by

$$
W=\left\{\zeta \in L^{2}((-1,1)): \int_{-1}^{1} e^{h(x)} \zeta^{2}(x) d x<\infty\right\}
$$

with inner product given as follows:

$$
\langle f, g\rangle=\int_{-1}^{1} e^{h(x)} f(x) g(x) d x \quad \text { for } \quad f, g \in W .
$$

The principal eigenvalue $\lambda_{1}$ is defined by the minimization problem

$$
\lambda_{1}=\min \left\{\int_{-1}^{1} w(x) e^{h(x)}\left(\zeta^{\prime}(x)\right)^{2} d x: \zeta \in H_{0}^{1}((-1,1)), \int_{-1}^{1} e^{h(x)} \zeta^{2}(x) d x=1\right\},
$$

and the other eigenvalues $\lambda_{j}, j=2,3, \cdots$, are determined by

$$
\lambda_{j}=\min \left\{\begin{array}{r}
\int_{-1}^{1} w(x) e^{h(x)}\left(\zeta^{\prime}(x)\right)^{2} d x: \zeta \in H_{0}^{1}((-1,1)), \\
\int_{-1}^{1} e^{h(x)} \zeta^{2}(x) d x=1, \\
\zeta \perp \zeta_{k}, k=1 \cdots, j-1
\end{array}\right\},
$$


where $\zeta \perp \zeta_{k}$ means orthogonality with respect to the inner product $\langle\cdot, \cdot\rangle$ defined by (5.11). Consequently, $\zeta \perp \zeta_{k}$ if and only if $\left\langle\zeta, \zeta_{k}\right\rangle=\int_{-1}^{1} e^{h} \zeta \zeta_{k} d x=0$. Because $w$ and $e^{h}$ are positive and smooth functions, these eigenvalues $\left\{\lambda_{j}\right\}$ are positive and the associated eigenfunctions $\left\{\zeta_{j}\right\}$ are well-defined.

To solve the equation (5.7), being same as the equation (5.8), Galerkin's method is used by setting the solution $\tilde{\psi}$ with the following form:

$$
\tilde{\psi}(x, t)=\sum_{j=1}^{\infty} a_{j}(t) \zeta_{j}(x)
$$

where $\zeta_{j}$ is the $j$ th eigenfunction of the operator $L$ with the associated eigenvalues $\left\{\lambda_{j}\right\}_{j=1}^{\infty}$ satisfying the weighted eigenvalue problem (5.10). Then

$$
\frac{1}{z D} \sum_{j=1}^{\infty} \frac{d a_{j}(t)}{d t} \zeta_{j}(x)=2 z^{2} e^{-h(x)}\left(w \phi_{0}{ }^{\prime}\right)^{\prime}(x)+\left(g_{n p}-g\right) \sum_{j=1}^{\infty} a_{j}(t) \lambda_{j} \zeta_{j}(x) .
$$

Fix $j \in \mathbb{N}$ arbitrarily. Taking the inner product (defined in (5.11)) of (5.12) and $\zeta_{j}$, the equation of $a_{j}$ is obtained as follows:

$$
\frac{1}{z D} \frac{d a_{j}}{d t}(t)=f_{j}+\left(g_{n p}-g\right) \lambda_{j} a_{j}(t) \text { for } t>0,
$$

where the $f_{j}$ are constants given by

$$
f_{j}=\left\langle 2 z^{2} e^{-h}\left(w \phi_{0}^{\prime}\right)^{\prime}, \zeta_{j}\right\rangle .
$$

Note that $\left\{\zeta_{j}\right\}_{j=1}^{\infty}$ is orthonormal with respect to the inner product (5.11). Moreover, the Schwartz inequality can be applied to (5.14) and implies

$$
\left|f_{j}\right| \leq 2 z^{2}\left\|e^{-h}\left(w \phi_{0}{ }^{\prime}\right)^{\prime}\right\|\left\|\zeta_{j}\right\|=2 z^{2}\left\|e^{-h / 2}\left(w \phi_{0}{ }^{\prime}\right)^{\prime}\right\|_{L^{2}} \equiv K_{2},
$$

where $K_{2}$ is a positive constant independent of $j$, and the norm $\|\cdot\|$ comes from the inner product $\langle\cdot, \cdot\rangle$ and is defined by $\|v\|=\langle v, v\rangle^{1 / 2}=\left\|e^{h / 2} v\right\|_{L^{2}}$ for $v \in L^{2}((-1,1))$. Notice that each $\zeta_{j}$ satisfies $\left\|\zeta_{j}\right\|=1$. On the other hand, the equation (5.13) can be solved and the explicit form of $a_{j}$ is obtained as follows:

$$
a_{j}(t)=\left[a_{j}(0)+\frac{f_{j}}{\left(g_{n p}-g\right) \lambda_{j}}\right] e^{z D\left(g_{n p}-g\right) \lambda_{j} t}-\frac{f_{j}}{\left(g_{n p}-g\right) \lambda_{j}} \quad \text { for } \quad t>0, j=1,2, \ldots
$$

Consequently, the solution $\tilde{\psi}$ has the following explicit form:

$$
\tilde{\psi}(x, t)=\sum_{j=1}^{\infty}\left\{\left[a_{j}(0)+\frac{f_{j}}{\left(g_{n p}-g\right) \lambda_{j}}\right] e^{z D\left(g_{n p}-g\right) \lambda_{j} t}-\frac{f_{j}}{\left(g_{n p}-g\right) \lambda_{j}}\right\} \zeta_{j}(x),
$$

for $x \in(-1,1)$ and $t>0$. Moreover, by (5.16), the assumption $g_{n p}>g$ implies that $\left|a_{j}(t)\right|$ may tend to infinity exponentially as $t$ goes to infinity, provided that the initial data $a_{j}(0)$ satisfies $a_{j}(0)+\frac{f_{j}}{\left(g_{n p}-g\right) \lambda_{j}} \neq 0$. Precisely speaking,

$$
\lim _{t \rightarrow \infty}\left|a_{j}(t)\right|=\infty \quad \text { if } \quad a_{j}(0)+\frac{f_{j}}{\left(g_{n p}-g\right) \lambda_{j}} \neq 0 .
$$


Note that each eigenvalue $\lambda_{j}$ is positive. Because $\tilde{\psi}(x, t)=\sum_{j=1}^{\infty} a_{j}(t) \zeta_{j}(x)$ and $\left\{\zeta_{j}\right\}_{j=1}^{\infty}$ is orthonormal with respect to the inner product $\langle\cdot, \cdot \cdot\rangle$ defined in (5.11), then $a_{j}(0)=\left\langle\tilde{\psi}_{0}, \zeta_{j}\right\rangle$, where $\tilde{\psi}_{0}=\left.\tilde{\psi}\right|_{t=0}$ is the initial data of $\tilde{\psi}$. Thus

$$
\lim _{t \rightarrow \infty} \int_{-1}^{1} \tilde{\psi}^{2} d x=\lim _{t \rightarrow \infty} \sum_{j=1}^{\infty}\left|a_{j}(t)\right|^{2}=\infty \quad \text { if } \quad\left\langle\tilde{\psi}_{0}, \zeta_{j}\right\rangle+\frac{f_{j}}{\left(g_{n p}-g\right) \lambda_{j}} \neq 0 \text { for some } j \in \mathbb{N},
$$

which implies that

$$
\lim _{t \rightarrow \infty} \int_{-1}^{1} \psi^{2} d x=\lim _{t \rightarrow \infty} \int_{-1}^{1} e^{2 h} \tilde{\psi}^{2} d x \geq B_{1}^{2} \lim _{t \rightarrow \infty} \int_{-1}^{1} \tilde{\psi}^{2} d x=\infty
$$

if $\left\langle\tilde{\psi}_{0}, \zeta_{j}\right\rangle+\frac{f_{j}}{\left(g_{n p}-g\right) \lambda_{j}} \neq 0$ for some $j \in \mathbb{N}$. Here (5.5) and (5.6) have been used to derive

$$
\psi^{2}=e^{2 h} \tilde{\psi}^{2} \geq B_{1}^{2} \tilde{\psi}^{2} .
$$

To solve the equation (5.4), let $\tilde{\varphi}(x, t)=w(x) \varphi(x, t)$ and transform the equation (5.4) into

$$
\frac{1}{z D w} \frac{\partial \tilde{\varphi}}{\partial t}=2 z w^{\prime \prime}+\left(g+g_{n p}\right) \frac{\partial^{2} \tilde{\varphi}}{\partial x^{2}} \quad \text { for } x \in(-1,1), t>0 .
$$

As for solving the equation (5.7), we may also apply the Galerkin method by setting $\tilde{\varphi}(x, t)=\sum_{l=1}^{\infty} b_{l}(t) \eta_{l}(x)$ and get

$$
\tilde{\varphi}(x, t)=\frac{2 z}{g+g_{n p}} \sum_{l=1}^{\infty} \frac{\left(w w^{\prime \prime}, \eta_{l}\right)}{\mu_{l}} \eta_{l}(x)+\sum_{l=1}^{\infty}\left[b_{l}(0)-\frac{2 z\left(w w^{\prime \prime}, \eta_{l}\right)}{\left(g+g_{n p}\right) \mu_{l}}\right] \eta_{l}(x) e^{-z D\left(g+g_{n p}\right) \mu_{l} t}
$$

for $x \in(-1,1)$ and $t>0$, where $\mu_{l}$ and $\eta_{l}$ are the $l$ th eigenvalue and eigenfunction of the following weighted eigenvalue problem:

$$
\left\{\begin{aligned}
-\eta^{\prime \prime}(x) & =\frac{\mu}{w(x)} \eta(x), \quad \text { for } x \in(-1,1) \\
\eta( \pm 1) & =0
\end{aligned}\right.
$$

As for the weighted eigenvalue problem (5.10), the problem (5.21) also has positive eigenvalues $\left\{\mu_{l}\right\}_{l=1}^{\infty}$ and eigenfunctions $\left\{\eta_{l}\right\}_{l=1}^{\infty}$ such that $0<\mu_{1} \leq \mu_{2} \leq \cdots$ and $\left\{\eta_{l}\right\}_{l=1}^{\infty}$ forms an orthonormal basis of the function space

$$
H=\left\{\eta \in L^{2}(-1,1): \int_{-1}^{1} \frac{1}{w(x)} \eta^{2}(x) d x<\infty\right\}
$$

with the inner product $(\cdot, \cdot)$ defined by

$$
(u, v)=\int_{-1}^{1} \frac{1}{w(x)} u(x) v(x) d x \quad \text { for } \quad u, v \in H .
$$

Furthermore, each $\left(\mu_{l}, \eta_{l}\right)$ satisfies

$$
\left\{\begin{array}{cc}
-\eta_{l}^{\prime \prime}(x)=\frac{\mu_{l}}{w(x)} \eta_{l}(x), & \text { for } x \in(-1,1), \\
\eta_{l}( \pm 1)=0, & l=1,2,3, \cdots
\end{array}\right.
$$


For the convergence of the series $\sum_{l=1}^{\infty} \frac{\left(w w^{\prime \prime}, \eta_{l}\right)}{\mu_{l}} \eta_{l}(x)$, the Bessel inequality and the fact that $\mu_{l} \geq \mu_{1}>0$ for $l \in \mathbb{N}$ are used to derive the inequalities

$$
\sum_{l=1}^{\infty}\left|\frac{\left(w w^{\prime \prime}, \eta_{l}\right)}{\mu_{l}}\right|^{2} \leq \frac{1}{\mu_{1}{ }^{2}} \sum_{l=1}^{\infty}\left(w w^{\prime \prime}, \eta_{l}\right)^{2} \leq \frac{1}{\mu_{1}{ }^{2}}\left\|w w^{\prime \prime}\right\|^{2},
$$

which imply that the series $\sum_{l=1}^{\infty} \frac{\left(w w^{\prime \prime}, \eta_{l}\right)}{\mu_{l}} \eta_{l}$ is convergent in the space $H$. Consequently,

$$
\lim _{t \rightarrow \infty} \tilde{\varphi}(x, t)=\frac{2 z}{g+g_{n p}} \sum_{l=1}^{\infty} \frac{\left(w w^{\prime \prime}, \eta_{l}\right)}{\mu_{l}} \eta_{l}(x) \equiv \tilde{\varphi}_{\infty}(x) \quad \text { for } x \in(-1,1),
$$

i.e.

$$
\lim _{t \rightarrow \infty} \varphi(x, t)=\frac{\tilde{\varphi}_{\infty}(x)}{w(x)} \quad \text { for } x \in(-1,1) .
$$

Combining (5.18) and (5.23), the instability of the system (2.43) with the zero Dirichlet boundary condition is proved. On the other hand, $z_{p}=-z_{n}=z>0, D_{p}=D_{n}=$ $D>0, g_{n p}<g_{n n}=g_{p p}=g$ also satisfies the condition of Corollary 4.2 which gives the asymptotic stability. Therefore, these results are summarized as follows.

Theorem 5.1. Suppose $z_{p}=-z_{n}=z>0, D_{p}=D_{n}=D>0$, and $g_{n n}=g_{p p}=g$. Then the system (2.43) with the zero Dirichlet boundary condition becomes unstable if $g_{n p}>$ $g$, but is asymptotically stable if $g_{n p}<g$.

Acknowledgment. The research of Lin is supported by NSC grant 100-2115-M002-007-MY3 of Taiwan and Taida Institute of Mathematical Sciences (TIMS). The research of Eisenberg is supported by the Bard Endowed Chair of Rush University. The authors also want to express their particular thanks to Chun Liu and Tzyy-Leng Horng for many useful discussions.

\section{REFERENCES}

[1] Z. Abbas, M. Gunnarsson, E. Ahlberg, and S. Nordholm, Corrected DebyeHuckel theory of salt solutions: Size asymmetry and effective diameters, J. Phys. Chem. B, 106(6), 1403-1420, 2002.

[2] V. Barcilon, D.P. Chen, R.S. Eisenberg, and J.W. Jerome, Qualitative properties of steadystate Poisson-Nernst-Planck systems: Perturbation and simulation study, SIAM J. Appl. Math., 57(3), 631-648, 1997.

[3] M.Z. Bazant, K. Thornton, and A. Ajdari, Diffuse-charge dynamics in electrochemical systems, Phys. Rev. E, 70, 021506-1-24, 2004.

[4] M.Z. Bazant, B.D. Storey, and A.A. Kornyshev, Double layer in ionic liquids: Overscreening versus crowding, Phys. Rev. Lett., 106(4), 046102, 2011.

[5] I. Borukhov, D. Andelman, and H. Orland, Steric effects in electrolytes: A modified PoissonBoltzmann equation, Phys. Rev. Lett., 79, 435, 1997.

[6] F. Brezzi, L.D. Marini, S. Micheletti, P. Pietra, R. Sacco, and S. Wang, Finite Element and Finite Volume Discretizations of Drift-Diffusion Type Fluid Models for Semiconductors, Centre National de la Recherche Scientifique Paris, France, Technical Report, 2002-1302, 2002.

[7] M. Burger, Inverse problems in ion channel modelling, Inverse Prob., 27, 083001, 2011.

[8] J. Che, J. Dzubiella, B. Li, and J.A. McCammon, Electrostatic free energy and its variations in implicit solvent models, J. Phys. Chem. B, 112(10), 3058-3069, 2008. 
[9] Y.G. Chen and J.D. Weeks, Local molecular field theory for effective attractions between like charged objects in systems with strong Coulomb interactions, PNAS, 103(20), 7560-7565, 2006.

[10] R.D. Coalson and M.G. Kurnikova, Poisson-Nernst-Planck theory approach to the calculation of current through biological ion channels, IEEE Transactions on Nanobioscience, 4, 81-93, 2005.

[11] B.Y. Dan, D. Andelman, D. Harries, and R. Podgornik, Beyond standard Poisson-Boltzmann theory: Ion-specific interactions in aqueous solutions, J. Phys.: Condensed Matter, 21(42), 424106, 2009.

[12] R. Eisenberg and D. Chen, Poisson-Nernst-Planck (PNP) theory of an open ionic channel, Biophys. J., 64, A22, 1993.

[13] R. Eisenberg, Atomic biology, electrostatics and ionic channels. New developments and theoretical studies of proteins, R. Elber. Philadelphia, World Scientific, 7, 269-357, 1996. Published in the Physics ArXiv as arXiv:0807.0715.

[14] B. Eisenberg and W. Liu, Poisson-Nernst-Planck systems for ion channels with permanent charges, SIAM J. Math. Anal., 38(6), 1932-1966, 2007.

[15] B. Eisenberg, Ions in fluctuating channels: Transistors alive, Fluc. Noise Lett., 11(1), 1240001, 2012.

[16] B. Eisenberg, Y. Hyon, and C. Liu, Energy variational analysis of ions in water and channels: Field theory for primitive models of complex ionic fluids, J. Chem. Phys., 133, 104104-1, 2010.

[17] B. Eisenberg, Mass action in ionic solutions, Chem. Phys. Lett., 511, 1-6, 2011.

[18] B. Eisenberg, Crowded charges in ion channels, in Advances in Chemical Physics, John Wiley and Sons, Inc., 77-223, 2011.

[19] B. Eisenberg, A Leading Role for Mathematics in the Study of Ionic Solutions, SIAM News, $45,11-12,2012$.

[20] B. Eisenberg, Life's Solutions. A Mathematical Challenge, Available on arXiv as http://arxiv.org/abs/1207.4737.

[21] D. Fraenkel, Simplified electrostatic model for the thermodynamic excess potentials of binary strong electrolyte solutions with size-dissimilar ions, Mole. Phys., 108(11), 1435-1466, 2010.

[22] D. Fraenkel, Monoprotic mineral acids analyzed by the smaller-ion shell model of strong electrolyte solutions, J. Phys. Chem. B, 115(3), 557-568, 2010.

[23] D. Gillespie, W. Nonner, and R.S. Eisenberg, Coupling Poisson Nernst Planck and density functional theory to calculate ion flux, J. Phys.: Condensed Matter, 14, 12129-12145, 2002.

[24] B. Hille, Ion Channels of Excitable Membranes, 3rd ed., Sunderland, MA: Sinauer Associates, Inc., 2001.

[25] T.L. Horng, T.C. Lin, C. Liu, and B. Eisenberg, PNP equations with steric effects: A model of ion flow through channels, J. Phys. Chem. B, 116(37), 11422-11441, 2012.

[26] J.J. Howard, J.S. Perkyns, and B.M. Pettitt, The behavior of ions near a charged walldependence on ion size, concentration, and surface charge, J. Phys. Chem. B, 114(18), 6074-6083, 2010.

[27] R.J. Hünter, Foundations of Colloid Science, Oxford University, New York, 1989.

[28] Y. Hyon, B. Eisenberg, and C. Liu, A Mathematical model for the hard sphere repulsion in ionic solutions, Commun. Math. Sci., 9(2), 459-475, 2011.

[29] J.N. Israelachvili, Intermolecular and Surface Forces, Academic, London, Second Edition, 1990.

[30] J.W. Jerome, Analysis of Charge Transport. Mathematical Theory and Approximation of Semiconductor Models, Springer-Verlag, New York, 1995.

[31] A.J. Jerri, The Shannon sampling theory-its various extensions and applications: A tutorial review, Proc. IEEE, 65, 1565-1596, 1997.

[32] B. Johannesson, Development of a generalized version of the Poisson-Nernst-Planck equations using the hybrid mixture theory: presentation of $2 D$ numerical examples, Transp. Porous Med., 85, 565-592, 2010.

[33] I. Kalcher, J.C.F. Schulz, and J. Dzubiella, Ion-specific excluded-volume correlations and solvation forces, Phys. Rev. Lett., 104(9), 097802, 2010.

[34] M.S. Kilic, M.Z. Bazant, and A. Ajdari, Steric effects in the dynamics of electrolytes at large applied voltages. II. Modified Poisson-Nernst-Planck equations, Phys. Rev. E, 75, 021503, 2007.

[35] H.J. Landau and H.O. Pollak, Prolate spheroidal wave functions, Fourier analysis and uncertainty II, Bell System Tech. J., 40, 65-84, 1961.

[36] J.E. Lennard-Jones and A.F. Devonshire, Critical and co-operative phenomena. III. A theory 
of melting and the structure of liquids, Proc. R. Soc. Lond. A, 169, 317-338, 1939.

[37] B. Li, Minimization of electrostatic free energy and the Poisson-Boltzmann equation for molecular solvation with implicit solvent, SIAM J. Math. Anal., 40, 2536-2566, 2009.

[38] B. Li, Continuum electrostatics for ionic solutions with nonuniform ionic sizes, Nonlin., 22, 811-833, 2009.

[39] E.H. Lieb and M. Loss, Analysis, Second Edition, Amer. Math. Soci., Providence, Rhode Island, 2001.

[40] B. Lu and Y.C. Zhou, Poisson-Nernst-Planck equations for simulating biomolecular diffusionreaction processes II: Size effects on ionic distributions and diffusion-reaction rates, Biophys. J., 100(10), 2475-85, 2012.

[41] P.A. Markowich, The Stationary Seminconductor Device Equations, Springer-Verlag, Vienna, 1986.

[42] P.A. Markowich, C.A. Ringhofer, and C. Schmeiser, Semiconductor Equations, Springer-Verlag, New York, 1990.

[43] E. Neher, Ion channels for communication between and within cells Nobel Lecture, Nobel Lectures, Physiology or Medicine 1991-1995. N. Ringertz. Singapore, World Scientific Publishing Co, 10-25, 1997.

[44] E. Neher and B. Sakmann, Single channel currents recorded from the membrane of denervated muscle fibers, Nature, 260, 799-802, 1976.

[45] J.H. Park and J.W. Jerome, Qualitative properties of steady-state Poisson-Nernst-Planck systems: Mathematical study, SIAM J. Appl. Math., 57(3), 609-630, 1997.

[46] V.A. Parsegian, Van der Waals Forces: A Handbook for Biologists, Chemists, Engineers, and Physicists, Cambridge University Press, 2006.

[47] Y. Rosenfeld, M. Schmidt, H. Lowen, and P. Tarazona, Fundamental measure free-energy density functional for hard spheres: Dimensional crossover and freezing, Phys. Rev. E, 55, 4245-4263, 1997.

[48] I. Rubinstein, Electro-Diffusion of Ions (Studies in Applied and Numerical Mathematics), SIAM, Philadelphia, PA, 1990.

[49] B. Sakmann and E. Neher, Single Channel Recording, New York, Plenum, 1995.

[50] Z. Schuss, B. Nadler, and R.S. Eisenberg, Derivation of PNP equations in bath and channel from a molecular model, Phys. Rev. E, 64(2-3), 036116, 2001.

[51] B. Nadler, Z. Schuss, A. Singer, and R.S. Eisenberg, Ionic diffusion through confined geometries: From Langevin equations to partial differential equations, J. Phys.: Condensed Matter, 16, S2153-S2165, 2004.

[52] S. Selberherr, Analysis and Simulation of Semiconductor Devices, Springer-Verlag, New York, 1984.

[53] A. Singer and J. Norbury, A Poisson-Nernst-Planck model for biological ion channels, an asymptotic analysis in a three-dimensional narrow funnel, SIAM J. Appl. Math., 70(3), 949-968, 2009.

[54] E. Stein and G. Weiss, Introduction to Fourier Analysis on Euclidean Spaces, Princeton University Press, 1971.

[55] G.C. Temes, V. Barcilon, and F.C. Marshall, The optimization of bandlimited systems, Proc. IEEE, 61, 196-234, 1973.

[56] G.M. Torrie and A. Valleau, Electrical double layers: 4. Limitations of the Gouy-Chapman theory, J. Phys. Chem., 86, 3251-3257, 1982.

[57] J.L. Vazquez, The Porous Medium Equation, Oxford University Press Inc., New York, 2007.

[58] J. Vincze, M. Valisko, and D. Boda, The nonmonotonic concentration dependence of the mean activity coefficient of electrolytes is a result of a balance between solvation and ion-ion correlations, J. Chem. Phys., 133(15), 154507-154506, 2010.

[59] G.W. Wei, Differential geometry based multiscale models, Bul. Math. Bio., 72, 1562-1622, 2010.

[60] G. Wei, Q. Zheng, Z. Chen, and K. Xia, Variational multiscale models for charge transport, SIAM Review, 54, 699-754, 2012.

[61] T. Xiao and X. Song, A molecular Debye-Huckel theory and its applications to electrolyte solutions, J. Chem. Phys., 135(10), 104104-104114, 2011.

[62] D.P. Chen, Nonequilibrium thermodynamics of transports in ion channels, in Progress of Cell Research: Towards Molecular Biophysics of Ion channels, M. Sokabe, A. Auerbach, and F. Sigworth (eds.), Elsevier, Amsterdam, 269-277, 1997.

[63] D. Chen, L. Xu, B. Eisenberg, and G. Meissner, Calcium Ion Permeation through the Calcium Release Channel (Ryanodine Receptor) of Cardiac Muscle, J. Phys. Chem., 107B, 91399145, 2003.

[64] D. Chen, L. Xu, A. Tripathy, G. Meissner, and R. Eisenberg, Permeation through the calcium release channel of cardiac muscle, Biophys. J., 73, 1337-1354, 1997. 
[65] D. Chen, L. Xu, A. Tripathy, G. Meissner, and B. Eisenberg, Selectivity and permeation in calcium release channel of cardiac muscle: Alkali metal ions, Biophys. J., 76, 1346-1366, 1999.

[66] D. Gillespie, Energetics of divalent selectivity in a calcium channel: The ryanodine receptor case study, Biophys. J., 94, 1169-1184, 2008.

[67] A. Singer, Z. Schuss, and R.S. Eisenberg, Attenuation of the electric potential and field in disordered systems, J. Stat. Phys., 119, 1397-1418, 2005. 